ARTICLE

\title{
Chromosome organization by a conserved condensin-ParB system in the actinobacterium Corynebacterium glutamicum
}

\author{
Kati Böhm', Giacomo Giacomelli1,2, Andreas Schmidt ${ }^{3}$, Axel Imhof (iD ${ }^{3}$, Romain Koszul (10) 4,5, \\ Martial Marbouty ${ }^{4 凶} \&$ Marc Bramkamp ${ }^{1,2}{ }^{\square}$
}

Higher-order chromosome folding and segregation are tightly regulated in all domains of life. In bacteria, details on nucleoid organization regulatory mechanisms and function remain poorly characterized, especially in non-model species. Here, we investigate the role of DNApartitioning protein ParB and SMC condensin complexes in the actinobacterium Corynebacterium glutamicum. Chromosome conformation capture reveals SMC-mediated longrange interactions around ten centromere-like parS sites clustered at the replication origin (oriC). At least one oriC-proximal parS site is necessary for reliable chromosome segregation. We use chromatin immunoprecipitation and photoactivated single-molecule localization microscopy to show the formation of distinct, parS-dependent ParB-nucleoprotein subclusters. We further show that $\mathrm{SMC} / \mathrm{Scp} A B$ complexes, loaded via ParB at parS sites, mediate chromosomal inter-arm contacts (as previously shown in Bacillus subtilis). However, the MukBEF-like SMC complex MksBEFG does not contribute to chromosomal DNA-folding; instead, this complex is involved in plasmid maintenance and interacts with the polar oriCtethering factor DivlVA. Our results complement current models of ParB-SMC/ScpAB crosstalk and show that some condensin complexes evolved functions that are apparently uncoupled from chromosome folding.

\footnotetext{
${ }^{1}$ Ludwig-Maximilians-Universität München, Fakultät Biologie, 82152 Planegg-Martinsried, Germany. ${ }^{2}$ Christian-Albrechts-Universität zu Kiel, Institut für allgemeine Mikrobiologie, 24118 Kiel, Germany. ${ }^{3}$ Biomedical Center, Protein Analysis Unit, Faculty of Medicine, Ludwig-Maximilians-Universität München, 82152 Planegg-Martinsried, Germany. ${ }^{4}$ Institut Pasteur, Unité Régulation Spatiales des Génomes, UMR3525, CNRS, F-75015 Paris, France. ${ }^{5}$ Institut Pasteur, Center of Bioinformatics, Biostatistics and Integrative Biology (C3BI), Paris, France. ${ }^{\circledR}$ email: martial.marbouty@pasteur.fr; bramkamp@ifam.uni-kiel.de
} 


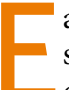
ach organism must complete genome replication and separation in the course of one cell cycle prior to cell division in concert with transcriptional processes. To this end, chromosomes are highly organized structures in terms of segregation and overall folding patterns ${ }^{1}$. The functional organization of bacterial genomes, structured into the nucleoid, has been predominantly investigated in a limited number of model species, e.g., Escherichia coli, Vibrio cholerae, Bacillus subtilis, or Caulobacter crescentus, revealing diverse levels of compaction and segregation strategies ${ }^{2-4}$.

ParABS systems and condensins are two (nearly) ubiquitous bacterial enzyme machineries that contribute to chromosome homeostasis. With a few exceptions among $\gamma$-proteobacteria, including E. coli, all branches of bacteria and several Archaea harbor parS sites that recruit partitioning protein $\operatorname{ParB}^{5}$. The ParABS system contains one or several parS sites usually in the vicinity to the chromosomal origin of replication (oriC). ParB proteins bind to these sequence-specific motives and form large nucleocomplexes by spreading and three-dimensional (3D)bridging between ParB dimers ${ }^{6-9}$, resulting in large chromosome interaction domains promoting encompassing the oriC, which have been revealed by chromosome conformation capture coupled to deep sequencing (Hi-C) for B. subtilis ${ }^{10}$. In an alternative model termed nucleation and caging, ParB nucleation at parS is stabilized by dynamic ParB dimer-dimer interactions and weak interactions with nonspecific DNA generating a scaffold for locally high ParB concentrations confined around pars $^{11}$. The ParB segregation is driven by a ParA ATPase, which binds nonspecifically to the nucleoid and is released from DNA upon ATP hydrolysis triggered by transient ParB interactions ${ }^{12,13}$. In the course of chromosome replication, ParB-oriC complexes act in combination with ParA as Brownian ratchets along dynamic DNA loci: slow ParA-DNA rebinding rates generate ParA gradients, which serve as tracks for directed movement of partition complexes away from their sisters $^{14-17}$. Perturbation of the system by placing parS sites at ectopic, oriC-distal regions can cause severe DNA-segregation phenotypes $^{18,19}$. To date, only few studies investigated the impact of chromosomal parS localization on DNA segregation and folding ${ }^{18-22}$.

In addition to ParABS systems, most bacteria harbor condensin complexes, members of the structural maintenance of chromosomes (SMCs) family of proteins found in all kingdoms of life ${ }^{23}$. In standard model organisms, condensins are equally essential for faithful chromosome segregation by compacting DNA into separate nucleoids ${ }^{24-26}$. The SMC/ScpAB complex is well-studied in $B$. subtilis, where it consists of two large SMC subunits and the kleisin ScpA associated with dimeric accessory protein ScpB that assemble into a ring-like structure ${ }^{27}$. A recent study suggests progressive extrusion of condensin-encircled DNA loops upon conformational changes in the SMC subunit, which leads to a gradual size increase of trapped DNA molecules ${ }^{28}$. The active process(es) driving DNA extrusion ${ }^{29,30}$ allow(s) for translocation along the chromosome with velocities of around $50 \mathrm{~kb} / \mathrm{min}$ (ref. ${ }^{31}$ ) and depend(s) on the ATPase activity of $\mathrm{SMC}^{32,33}$. To be loaded on parS sites, SMC/ $\mathrm{Scp} A B$ complexes necessitate $\mathrm{ParB}^{20,22,34,35}$. They redistribute to distant chromosomal regions, promoting the co-alignment of the right and left replichores ${ }^{10,21,22,31,36}$. In sharp contrast with SMC/ $\mathrm{ScpAB}$, the E. coli condensin MukBEF does not promote the coalignment of chromosomal arms $\mathrm{s}^{37,38}$, but facilitate cis-structuration by establishing long-range contacts between loci belonging to the same replichores from stochastically positioned chromosomal loci (except for the ter region containing the replication terminus $)^{38,39}$. Despite the importance of condensins in chromosome organization, the role of SMC homologs besides the model species $B$. subtilis, C. crescentus, and E. coli remain largely unexplored.
These species all contain a single condensin complex, yet a broad range of bacteria possesses combinations of $\mathrm{SMC} / \mathrm{ScpAB}$ and MksBEFG (MukB-like SMC), for which functional characterizations are non-existent to date ${ }^{40}$. Current work in bacteria and in eukaryotes convey the general assumption that all SMCs are likely to play role(s) in chromosome organization. In bacteria, it remains unknown why some species harbor more than one type of condensin, and whether and how they would work in concert with each other and coordinate with systems such as ParABS.

In this work, we used a combination of high-resolution microscopy and genomic chromosome conformation capture $(3 \mathrm{C} / \mathrm{Hi}-\mathrm{C})^{36}$ to unveil the global organization of the diploid Corynebacterium glutamicum genome. C. glutamicum is a polar growing actinobacterium, whose genome encodes both SMC/ $\mathrm{ScpAB}$ and MksBEFG. In this species, the two oriCs are continuously associated with the polar scaffold protein DivIVA, whereas newly replicated sister oriCs segregate towards division septa via the ParABS system ${ }^{41-43}$. In contrast to B. subtilis, $C$. glutamicum $\mathrm{Par} A \mathrm{~B}$ are by themselves crucially important drivers of reliable nucleoid separation prior to cell division, where ParAB deletions yield in $20 \%$ of anucleate cells ${ }^{44-46}$. Here, analyses of chromosomal ParB-binding patterns evince ten redundant parS sites, which mediate ParB subcluster formation at oriC. A single parS site maintains ParB propagation over $32 \mathrm{~kb}$ neighboring regions and is sufficient to promote the SMC-dependent alignment of the two chromosomal arms. Hi-C also reveals SMCdependent long-range contacts surrounding oriC. In contrast to SMC, we show that the polar positioned MksBEFG condensin acts mostly on plasmid transmission to daughter cells, without obvious influence on nucleoid architecture.

\section{Results}

Chromosome segregation is governed by ten oriC-proximal parS sites. Previous studies on C. glutamicum chromosome partitioning have revealed two stable ParB-oriC clusters at each cell pole, whereas newly replicated origins are segregated towards a division septum formed at midcell ${ }^{41}$. In B. subtilis, C. crescentus and Pseudomonas aeruginosa ParAB-mediated chromosome segregation and folding depends on parS sites ${ }^{18,19,21}$. In C. glutamicum, parS positions have not been characterized yet. Four to eight parS sites were predicted earlier in Corynebacterineae ${ }^{5}$. A BLAST analysis pointed at ten $B$. subtilis-like 16 bp consensus sequences in C. glutamicum, localized in one cluster within a $35 \mathrm{~Kb}$ region at $73 \mathrm{~Kb}$ from oriC $(1 \%$ of the $3.21 \mathrm{Mb}$ chromosome; Fig. 1a). Out of the ten parS sites, only the furthest from oriC ( $p a r S 1)$ lies within a coding sequence ( $\operatorname{trp} C F)$. All other parS sequences (labeled parS 2-10) were positioned within intergenic regions. Degenerated parS sequences with at least three base-pair mismatches were also identified further away from oriC, e.g., $5^{\prime}$ of cg0146 or within the fusA and cg1994 coding region. To test whether these putative parS were responsible for the recruitment of ParB, chromatin immunoprecipitation (ChIP) of ParB was performed with a strain harboring a mCherry-tagged version of the native ParB (note that all mutant strains used in this study derive from clean allelic replacements and have, unless otherwise noted, a wild-type-like phenotype). Distinct and very reproducible enrichment signals were obtained at the ten pars sites close to oriC (parS1-10 at $3.16 \mathrm{MB}$ ) (Fig. 1a), whereas the degenerated parS sequences failed to recruit ParB. Additional smaller peaks were identified at highly transcribed DNA regions, in particular at ribosomal genes, transfer RNA gene clusters, and at all of the ribosomal RNA operons (Fig. 1a). Magnification of the oriC region reveals three distinct ParB propagation zones overlapping with parS1-4, parS5-8, and parS9-10, respectively (Fig. 1b). Remarkably, those three regions seem to recruit 
a
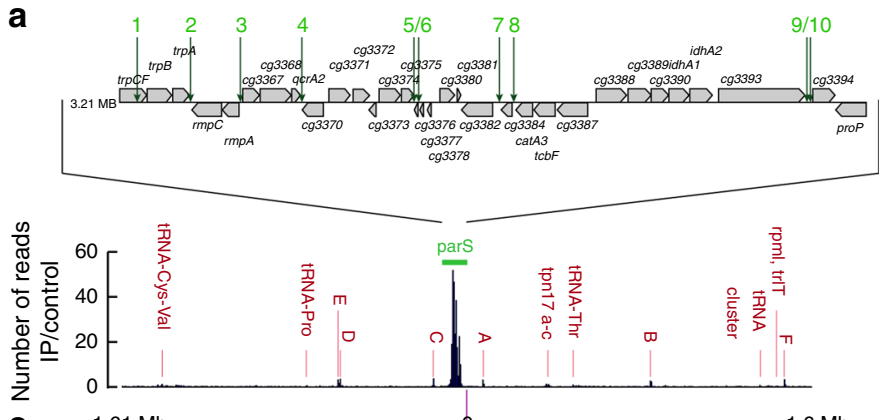

C

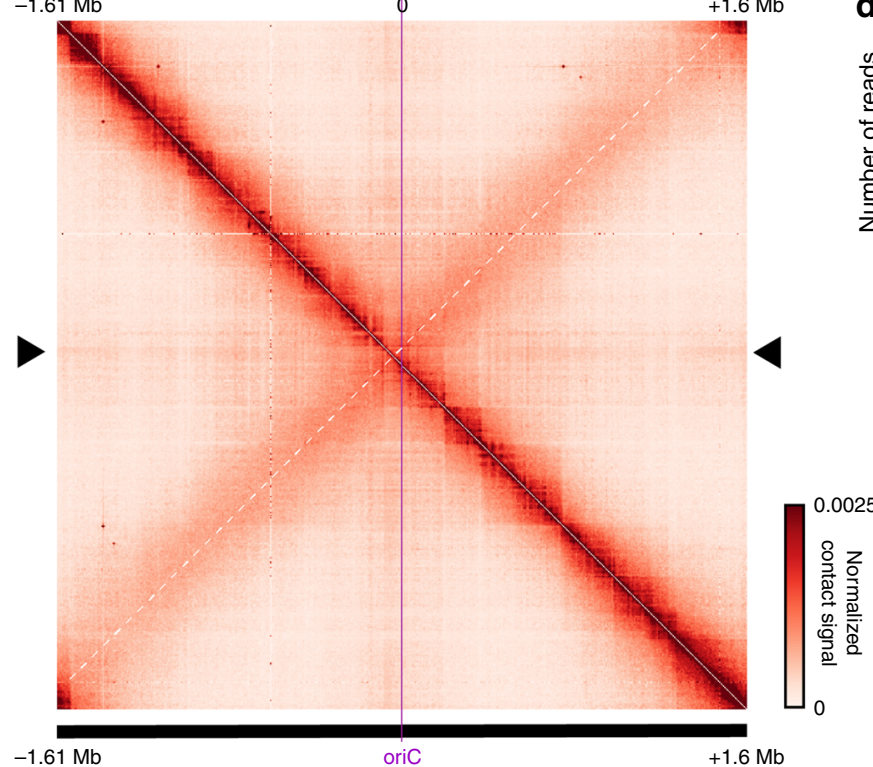

b

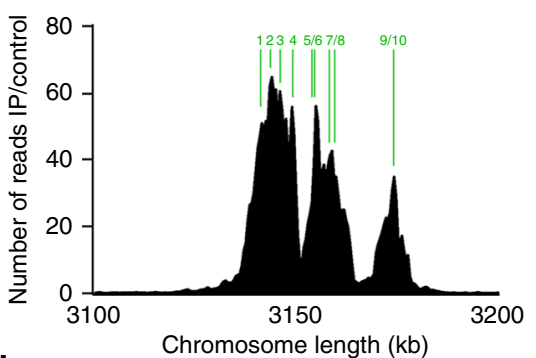

d
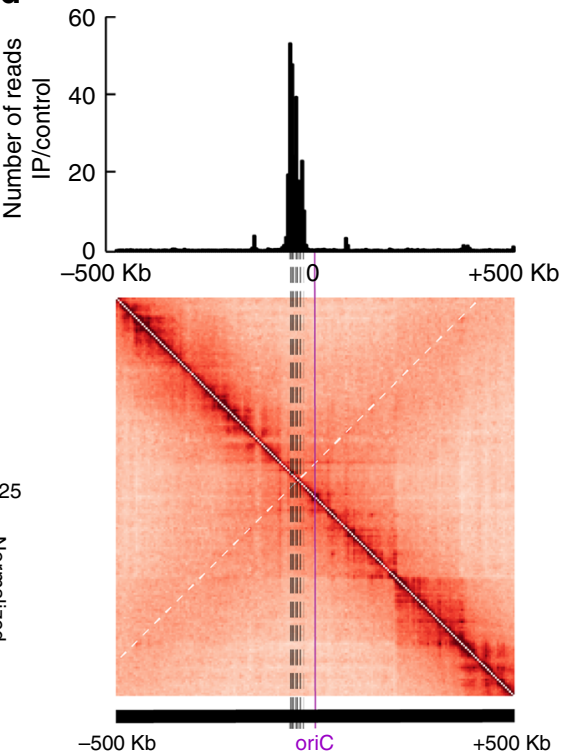

Fig. 1 Chromosome organization hub at oriC domain in C. glutamicum. a Top: genomic region including ten parS sites of $C$. glutamicum with 16 bp consensus sequences. Below: ChIP-seq data on ParB-mCherry DNA-binding protein confirm parS sites shown above. Exponentially growing C. glutamicum parB::parB-mCherry cells (CBK006) were used for in vivo anti-mCherry ChIP-seq experiments. Shown is the ratio of ChIP signal relative to the input (foldenrichment IP/control) in $5 \mathrm{~Kb}$ bins in linear scale along the chromosome with an $x$-axis centered at oriC. Red labels indicate minor enrichment signals at highly transcribed regions, such as rRNA operons (letters A-F). b ParB-ChIP-seq enrichment encompassing 3.1-3.2 Mb genomic region; parS sites 1-10 are indicated (green lines). c Normalized genomic contact map derived from asynchronously grown cells (fast growth, growth rate $(\mu) \geq 0.6 \mathrm{~h}^{-1}$, exponential phase). $X$ - and $Y$-axes indicate chromosomal coordinates binned in $5 \mathrm{~Kb}$; oriC-centered (purple bar-coordinate 0 ). Color scales, indicated beside the contact map, reflect contact frequency between two genomic loci from white to red (rare to frequent contacts). White dashed line on the contact matrix indicate the mean signal of the secondary diagonal and black triangles on the side of the contact matrix indicate the "cross like" signal. d Structural chromosome organization of the oriC region. Magnification of contacts within $500 \mathrm{~Kb}$ surrounding oriC; oriC is indicated as a purple line and parS sites are indicated by dashed lines. ParB-enrichment zones at parS are shown above the contact map (ChIP signal relative to the input in $5 \mathrm{~Kb}$ bins). White dashed line on the contact matrix indicate the mean signal of the secondary diagonal.

decreasing amounts of ParB, from parS1-4 (most enriched) to parS9-10 (less enriched). As all parS are identical in sequence, differences in ParB recruitment might result from the number and distance of parS sequences in the context of the overall nucleoid folding patterns at the oriC region.

Higher-order organization of the C. glutamicum chromosome. In B. subtilis, SMC-mediated chromosome folding initiates at ParB-parS clusters surrounding the oriC, bridging the two replichores with each other ${ }^{10,21}$. To characterize whether C. glutamicum parS sites play a similar role in the overall organization of the chromosome, we applied a Hi-C-like approach ${ }^{10,47}$ to exponentially growing wild-type cells (Methods). The genome-wide contact map, displaying the average contact frequencies between all $5 \mathrm{~Kb}$ segments of wild-type chromosomes (Fig. 1c) displayed the following 3D features. First, a strong and broad diagonal reflecting frequent local contacts between adjacent loci and observed in all Hi-C experiments. Second, chromatin interaction domains (CIDs), i.e., self-interacting regions previously described in C. crescentus and other species ${ }^{10,21,36,38,48}$ (Fig. 1c and Supplementary Fig. 1) (11 domains detected at a $200 \mathrm{~Kb}$ resolution). In C. glutamicum, 6 out of 11 boundaries are associated with high transcriptional activity or gene lengths (Supplementary Fig. 1A). The Hi-C signal did not present overall a clear correlation with transcriptional activity (Supplementary Fig. 1B). Other roadblocks like nucleoid-associated proteins might play a major role in confining chromosomal interaction domains. Third, a secondary diagonal perpendicular to the main one and extending from the ori-proximal, $35 \mathrm{~Kb}$ parS cluster (Fig. 1d, white dashed line) down to the replication terminus. This structure shows that the two replichores are bridged over their entire length, as in B. subtilis ${ }^{10,21,36}$. Interestingly, this secondary diagonal also displays discrete, long-range contact enrichments (Fig. 1c), which may reflect bridging of the two chromosomal arms at specific locations. Finally, the contact map also displays a faint, crossshaped signal corresponding to contacts between the ori region and the rest of the chromosome (Fig. 1c, dark triangle on the sides of the contact matrix), as in B. subtilis ${ }^{21}$. These contacts 
might be due to the segregation and translocation of the ParBoriC complex along the nucleoid during segregation when oriCs reposition at midcell. This signal is also maximal at the parS cluster and not at oriC locus (Fig. 1c, d). This observation reinforces the fact that the parS cluster is at the tip of Corynebacterium chromosome fold and is one of the main actors of chromosome segregation. A similarity matrix between the different constructed $\mathrm{Hi}-\mathrm{C}$ matrices was calculated (Supplementary Fig. 2A) and flow cytometry was performed with all samples used for Hi-C analyses to control for chromosome number and potential replication differences (Supplementary Fig. 2B-D).

A single parS site is sufficient to maintain chromosome architecture. As all parS sites are in close proximity on the $C$. glutamicum chromosome, we tested the importance of ParB-parS complex titration for the overall chromosome organization. Cells with chromosomes carrying the single parS1 site (for parS mutations, see Supplementary Fig. 3A) grow and divide like wildtype cells (Supplementary Figs. $2 \mathrm{~A}$ and $3 \mathrm{~B}$ ). However, the removal of all ten parS sites resulted in a cell length phenotype (Supplementary Fig. 3B) and 29\% DNA-free mini-cells, hinting to a nucleoid segregation defect similar to the $\triangle$ parB phenotype (Fig. 2a and Supplementary Table 1). We further analyzed ParB localization in mutant strains carrying either a single or no parS site. First, if only parS1 is present, cellular localization of fluorescent ParB-mCherry foci is similar to wild type, positioning at cell poles and migrating to the newly formed septa ${ }^{41}$ (Fig. 2b). Interestingly, the combination of a single parS site with ParBeYFP resulted in 7\% anucleate mini-cells (Supplementary Fig. 3C and Supplementary Table 1), reflecting functional constraints of the ParB-eYFP fusion in the presence of only one parS site. Therefore, the high number of chromosomal parS sites likely evolved to improve the robustness of the segregation machinery. ParB ChIP-quantitative PCR (qPCR) signals of locus parS1 were similar in both wild-type and mutant strains (Fig. 2c). ParB spreading around the single parS site was characterized through ChIP-seq analysis (Fig. 2d and Supplementary Fig. 4), where ParB binding was maximum within $2 \mathrm{~Kb}$ windows on both sides of pars, while extending up to $16 \mathrm{~Kb}$ on either side. However, redundancy of parS sites is not restricted to parS1 in C. glutamicum, as exemplified by the analysis of the single parS10, which was equally sufficient for wild-type-like growth and morphology (Supplementary Fig. 3D-E). A single parS10 site recruits ParB exclusively within the third nucleation zone encompassing $26 \mathrm{~Kb}$ (Supplementary Fig. 3A, F). We next investigated the role of parS sites and $\mathrm{ParB}$ in the overall chromosome folding by performing $\mathrm{Hi}-\mathrm{C}$ in mutants (Fig. 2e, f). The absence of either ParB or of all parS sites led to the disappearance of the secondary diagonal. In addition, the cross-shaped pattern reflecting contacts between the ori and the whole chromosome disappears in those mutants, also illustrated by the ratio between wild-type and mutant contact maps (Fig. 2f). This result shows that parS sites and ParB are two major structural components of chromosome organization and act in the same pathway to recruit downstream factors that fold the chromosome emanating from the pars cluster, and bridge the two chromosomal arms together down to the replication terminus region. The contact map of the strain deleted for parS2-10, but carrying a single parS1, maintains a secondary diagonal, showing that parS1 alone is sufficient to ensure the loading of ParB and the overall folding of the chromosome (Fig. 2e, f). However, some differences appeared between the wild type and the single parS1 site contact maps. In the mutant, the large domain surrounding the oriC shows minor differences in the contact maps compared with wild type, suggesting that a single parS site is not sufficient to fully restore the complexity of Corynebacterium chromosome ori folding (Fig. 2e, f).
The single parS site was then repositioned at different genomic regions. Cells harboring an ectopic, single parS site at $9.5^{\circ}, 90^{\circ}$, $180^{\circ}$, or $270^{\circ}$ positions were viable (Supplementary Fig. $5 \mathrm{~A}-\mathrm{C}$ ). Unlike cells harboring parS1 at its original position, ParB-parS complexes distribute virtually randomly along the longitudinal cell axis in all of these mutants (Supplementary Fig. 5C), resulting in $\sim 25 \%$ anucleate cells (Supplementary Table 1). Therefore, parS shifts result in nucleoid segregation defects. The number of ParB foci nevertheless correlates well with cell length (Supplementary Fig. 5D), excluding replication initiation deficiencies. ParB binding to a pars sequence positioned at the $90^{\circ}$ chromosomal position (locus cg0904, strain CBK042) was identified in a $9 \mathrm{~Kb}$ range on either side of parS (Supplementary Figs. 4A, 5E), approximately half the ParB-propagation distance determined for cells harboring one parS at its native locus. We also analyzed the mutant harboring parS at $90^{\circ}$ chromosomal position by $\mathrm{Hi}-\mathrm{C}$ (CBK037). The contact map of this mutant displays a "bow shape" or a hairpin motif at the position of the aberrant parS sequence (Supplementary Fig. 6), reminiscent to the one observed in B. subtilis at the level of the ori-distal parS site and pointing a local folding of the chromosome (Supplementary Fig. 6A). Collectively, these results show a redundancy of parS sites, with an optimal function confined to the oriC-proximal region.

PALM identifies ParB subclusters. To directly characterize oriC domain compaction via ParB, we applied photoactivated localization microscopy (PALM) to visualize individual ParBPAmCherry molecules with nanometer resolution $(\sim 20 \mathrm{~nm}$ localization precision). PALM revealed distinct ParB-dense regions at cell poles and quarter position regions, similar to foci observed via diffraction-limited epifluorescence microscopy (Fig. 2g). These ParB-enriched regions (macroclusters) display heterogeneous densities, with a variable number of higher density zones within subclusters. Macro- and subclusters have been identified via the OPTICS algorithm that orders data points according to their spatially closest neighbors for identification of clustering structures ${ }^{49,50}$ (see Methods and Supplementary Fig. 7A) and analyzed in strains harboring a single, two, or all the parS sites (Fig. $2 \mathrm{~g}$ and Supplementary Fig. 7B). We define a macrocluster as 32 individual events being localized within a maximum distance of $50 \mathrm{~nm}$ for macroclusters and $35 \mathrm{~nm}$ for subclusters, yielding in cluster numbers that are in line with ParB epifluorescence data and oriC numbers determined by flow cytometry (Supplementary Table 2). It is noteworthy that high chromosome numbers promote inter-molecular oriC colocalization in fast-growing cells. For more accurate cluster estimations, PALM analysis was performed using slow-growing cells resulting in significantly fewer ParB macroclusters per cell (Supplementary Fig. 7C $)^{41}$. As segregation of oriC complexes might alter their DNA compaction, we focused on the two largest macroclusters per cell, stably tethered at cell poles. Although this is not a direct measurement of the number of ParB nucleation points (parS), a strain with a higher number of parS sites can be expected to result in higher ParB density variability when compared with one which contain a single or no nucleation point. The amount of ParB contained within each macrocluster in wild type is significantly higher than in cells containing the single parS1 site (Fig. 2h), in agreement with the ParB deposition observed via ChIP-seq. A parallel between PALM and ChIP-seq can also be drawn with respect to the number of subclusters per macrocluster, with a higher number of subclusters in the wild type that accordingly harbors three ParB nucleation zones along the parS cluster compared with the single parS site forming only one zone (Fig. 2h). Absence of all parS sites likewise results in a significant 
a
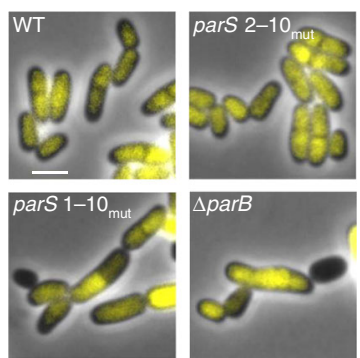

C

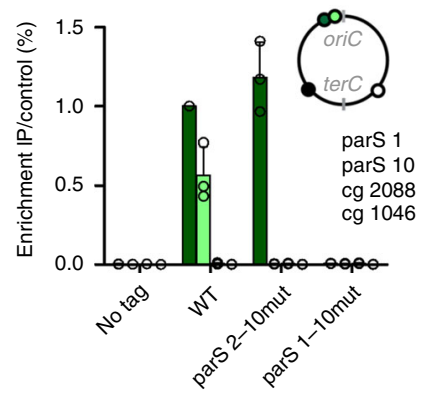

b

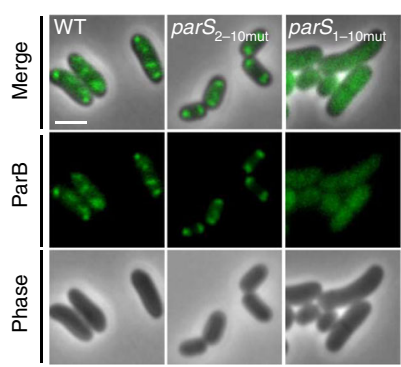

d

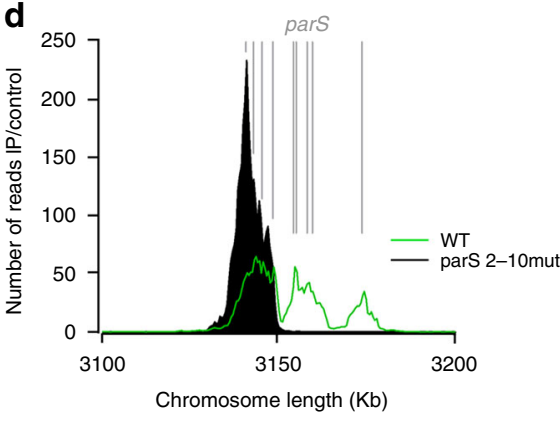

e

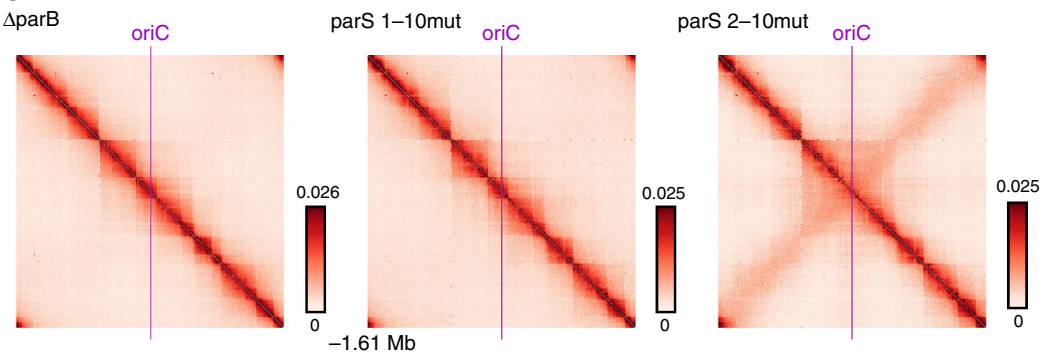

f

oriC

oriC
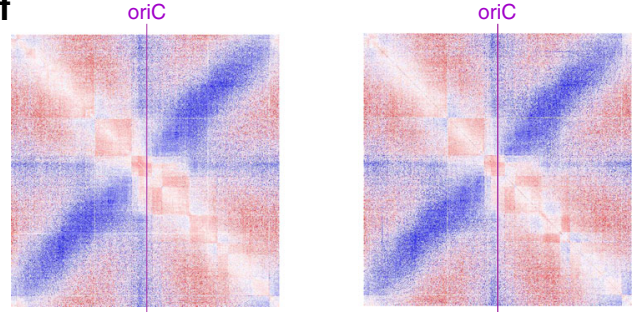

oriC

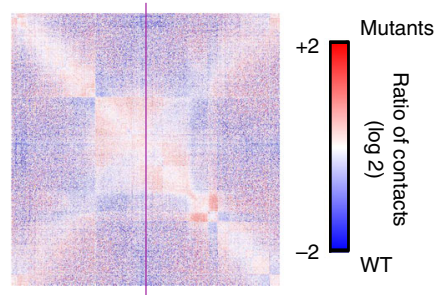

g

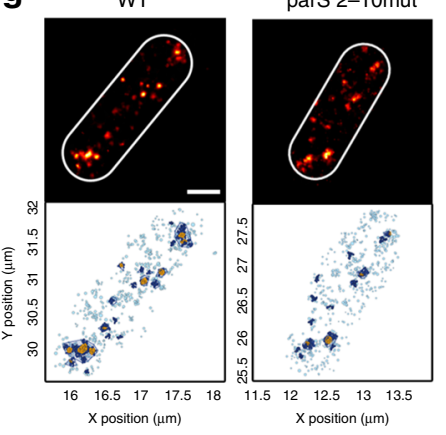

h

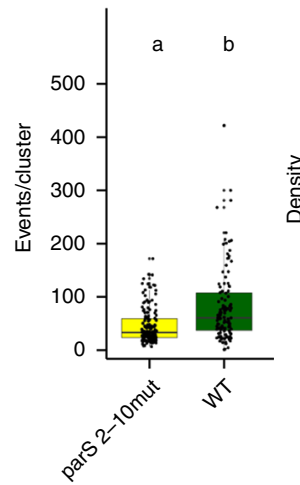

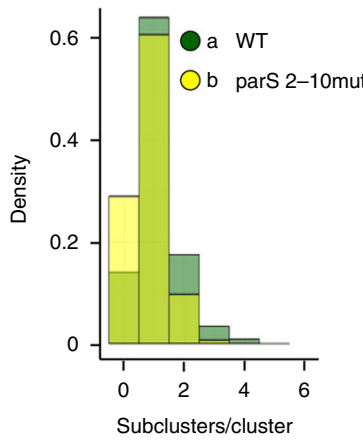

reduction of ParB macrocluster size and subcluster numbers compared with wild type (Supplementary Fig. 7D). These differences were not observed when comparing cells harboring all or two parS sites (parS1,10), which harbors two distinct ParB nucleation regions surrounding parS1 and parS10 (Supplementary Figs. 4A, 5F, and 7E). These observations could explain the differences observed between contact matrices of wild type and $\Delta$ parS 2-10 strains, and the higher structuring of the oriC domain when only one parS1 site is present. We therefore conclude that the architecture of the C. glutamicum partition complex is dependent on parS, and that ParB-parS nucleoprotein complexes are visible as individual subclusters. 
Fig. 2 A single parS site mediates chromosome folding. a One parS site is necessary and sufficient for wild type-like morphology and nucleoid segregation. Phase-contrast images of exponentially grown cells harboring either all (WT), one (parS 2 -10mut, $C B K 023$ ), or none (parS 1 -10mut, $C B K 024)$ parS site(s), or lacking parB ( $\triangle$ parB, CDC003) are shown. DNA is stained with Hoechst (yellow). Scale bar, $2 \mu \mathrm{m}$. b Fluorescence microscopy analysis of parB: :parB$m$ Cherry (shown in green) in wild type (CBK006), parS 2-10mut (CBK027), and parS 1-10mut backgrounds (CBK028). Absence of parS leads to diffuse cellular ParB localizations. Scale bar, $2 \mu \mathrm{m}$. c ChIP-qPCR for strains described before, normalized to wild-type parS1 signal (mean + SD, $n=3$ ). d ChIP-seq of $C$. glutamicum parB::parB-mCherry parS 2 -10mut (black) at a 3.1-3.2 Mb chromosomal range. Wild-type-like propagation (green) of ParB protein around parS1-4; $0.5 \mathrm{~Kb}$ bin size. Location of parS sites present in wild type or mutant sequences are indicated (gray lines). e Normalized contact maps of $\Delta$ parB, parS $\mathrm{S}_{1-10 \mathrm{mut}}$ and parS 2 -10mut mutants centered at oriC (CDC003, CBK024, and CBK023). Color codes as in Fig. 1. f Differential maps correspond to the log2 of the ratio (wild-type norm/mutant norm); color scales indicate contact enrichment in mutant (blue) or wild type (red) (white indicates no differences between the two conditions). $\mathbf{g}$ Single-molecule localization microscopy of representative wild-type and parS 2-10mut cells (CBK009 and CBK029). Top: Gaussian rendering of ParB-PAmCherry signals ( $0.71 \mathrm{PSF}, 1 \mathrm{px}=10 \mathrm{~nm}$ ), below: color-coded representation of ParB-PAmCherry events within corresponding cells ${ }^{49}$; all events (light blue), macroclusters (dark blue) and subclusters (yellow) are indicated. Scale bar, $0.5 \mu$ m. See Methods and Supplementary Fig. 7 for details. h Comparison of ParB-PAmCherry cluster properties. Only the two biggest clusters per cell were taken into account for analyses; significant differences between conditions are indicated by small letters above datasets. Left: events per macrocluster, medians are indicated as solid lines, and whiskers mark 1.5 IQRs (interquartile ranges); clusters wild type $_{n} n=130$, clusters pars2-10mut $_{n} n=143$. Right: subcluster numbers per macrocluster shown as overlay bar chart for both strains. Number of subcluster per macrocluster (two-tailed Kruskal-Wallis rank-sum test: $\chi^{2}=12.284, \mathrm{df}=1, p=0.0004569$ ) and macroclusters size (two-tailed Kruskal-Wallis rank-sum test: $\chi^{2}=27.582, \mathrm{df}=1, p=1.506 \mathrm{e}-07$ ) differ significantly between. Source data are provided as a Source Data file.

C. glutamicum harbors two paralogs of condensin complexes. In bacteria, the condensin paralog complexes $\mathrm{SMC} / \mathrm{Scp} A B$ and, in E. coli and other enterobacteria, MukBEF, are key factors of chromosome folding ${ }^{10,21,36,38}$. MksBEF (for MukBEF-like SMC) is another condensin occasionally found in bacterial genomes ${ }^{40}$, whose role(s) remain(s) obscure. A sequence homology search of the C. glutamicum genome pointed at the presence of both SMC/ ScpAB and MksBEF. The SMC/kleisin is encoded by genes cg2265 $(s m c), \operatorname{cg} 1611(\operatorname{scp} A)$, and $\operatorname{cg} 1614(\operatorname{scp} B)$ (Fig. 3a), whereas the Mks complex is encoded on a widely conserved operon ${ }^{40}$ and comprises genes cg3103-cg3106 ( $m k s G B E F$ ) (Fig. 3a), including MksG, which was being suggested to act in complex with MksBEF $^{40}$.

To characterize condensin complex formation in vivo, mass spectrometry of anti-mCherry pulldown experiments using SMCmCherry and MksB-mCherry as baits of whole-cell lysates were performed. Wild-type-like growth of corresponding strains and stability of SMC and MksB fluorescent fusions were confirmed (Supplementary Fig. 8A, C). Kleisin subunit ScpA and ScpB coprecipitated significantly with SMC compared with the negative control containing free mCherry, whereas subunits MksF and MksE, but not MksG, were substantially enriched in the MksB pulldown experiments (Supplementary Fig. 8D). ParB, which mediates SMC loading onto DNA in B. subtilis and $S$. pneumonia $20,34,35$, was not immunoprecipitated with SMC in any of the experiments. Bacterial two-hybrid analyses confirmed mass spectrometry results, pointing at the formation of $\mathrm{SMC} /$ $\mathrm{Scp} A B$ and MksBEF complex (Fig. 3b, c). No significant interactions between $S M C / S c p A B$ and $P a r B$ were detected, and we observed ScpA-ScpA self-interaction signals well above background. Moreover, MksG connects to the MksBEF complex via interaction with MksF, whereas the condensin complex subunits MksF and MksG further interact with the C. glutamicum polar scaffold protein DivIVA. Proteomics analysis failed to identify DivIVA in any immunoprecipitation. A possible reason for this is the high degree of DivIVA oligomerization by molecular bridging at cell membranes ${ }^{51,52}$.

SMC-mediated cohesion of chromosomal arms. We aimed to characterize C. glutamicum condensin SMC/ScpAB. Mutation of the SMC/ScpAB complex causes a conditionally lethal phenotype due to chromosome mis-segregation in $B$. subtilis ${ }^{25}$. In sharp contrast, a smc deletion in C. glutamicum did not result in either growth defects, DNA-segregation defects, or aberrant cell length distributions and morphologies compared with the wild type in minimal or complex media (Supplementary Fig. 9A, B and Supplementary Table 1). Nonetheless, the combination of genetic backgrounds parB: :parB-eYFP and $\triangle s m c$ yield a minor fraction of anucleate cells (4-5\%) (Supplementary Fig. 9A, C and Supplementary Table 1), indicating that SMC and ParB function in the same pathway and with ParB being epistatic to SMC. Hence, a functional interaction of SMC and ParB proteins regulating chromosome organization is likely. To further determine cellular localization of $\mathrm{SMC} / \mathrm{ScpAB}$ complexes, a strain harboring a fluorescently tagged version of core subunit SMC was imaged, revealing the formation of SMC clusters along the entire longitudinal axis of the cell (Fig. 3d). Clusters of SMC and ParB investigated in a strain carrying both labeled complexes (parB: :parB-mNeonGreen smc: smc-mCherry) are often proximal but do not always colocalize, whereas the foci numbers correlate with cell length (Fig. 3e). Up to eight SMC-mCherry foci were counted per cell. On average, cells contained fewer SMC-foci than ParB nucleoprotein complexes (Supplementary Fig. 8B). To further characterize the role of SMC, we generated Hi-C contact maps of the mutant (Fig. 3f). Deletion of $s m c$ abolishes the secondary diagonal in the maps (Fig. 3f), indicating that SMC and ParB function in the same pathway and have a synthetic phenotype concerning the cohesion of the two chromosomal arms. The combination of $s m c$ and parB mutations mimics a parB phenotype (Supplementary Fig. S9A, D), again resulting in the loss of contacts between chromosomal arms and further in the loss of the segregation signal described before (Fig. 3f, g). However, $\triangle$ parB and $\Delta s m c$ contact maps display different patterns along the main diagonal, suggesting that those two proteins affect differently chromosome architecture of C. glutamicum (Supplementary Fig. 9E). Therefore, it appears that an interplay of SMC/ScpAB with ParB is responsible for replichore cohesion in C. glutamicum, similar to B. subtilis and C. crescentus each harboring only one type of condensin complex ${ }^{10,21,22,36}$.

ParB-dependent SMC recruitment to chromosomal loading sites. As cellular SMC-mCherry signal hinted to distinct agglomeration clusters along the C. glutamicum chromosome, we investigated its putative binding sites via ChIP-seq. A small enrichment in SMC deposition was detected at and around the parS1-10 cluster (Fig. 4a), which disappears upon parB or parS deletion (Fig. 4a and Supplementary Figs. 4 and 10A, B). In addition, comparably minor enrichment signals are present throughout the chromosome, which partially coincide with genomic loci of high transcriptional activity. Distinct SMC- 
a

$1.5 \mathrm{ME} \prod_{c g 1612}^{\text {pldp scpA }}$

$2.15 \mathrm{MB}$

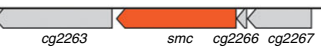

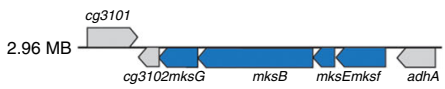

b

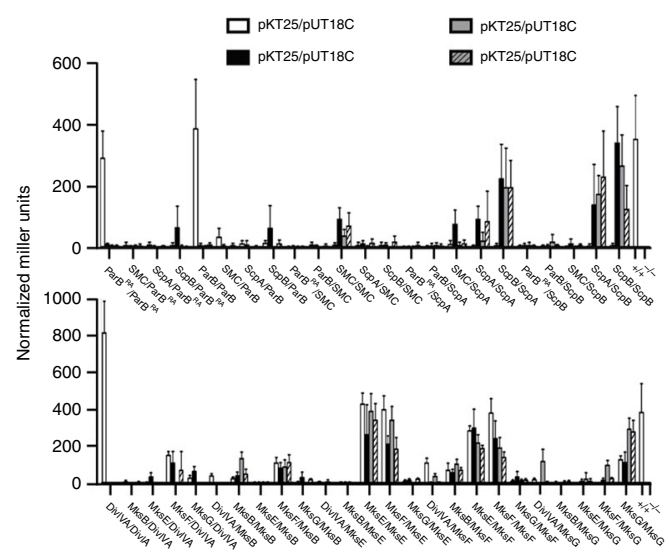

C
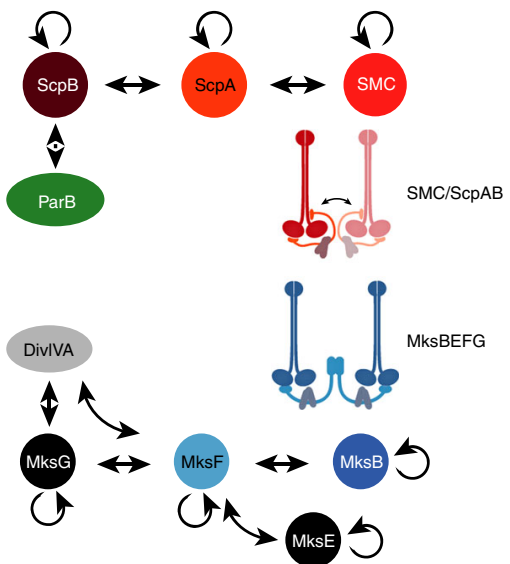

d
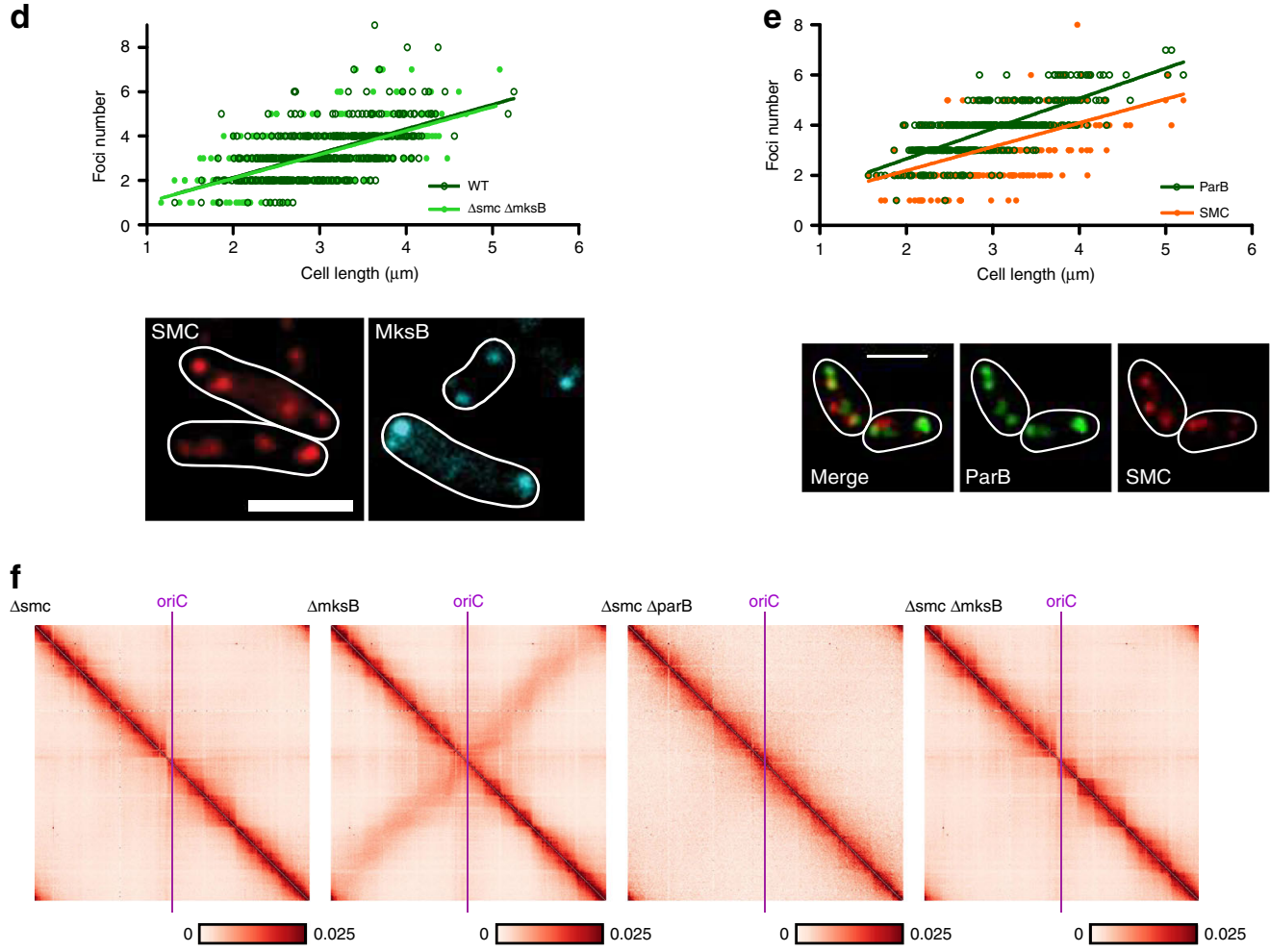

g
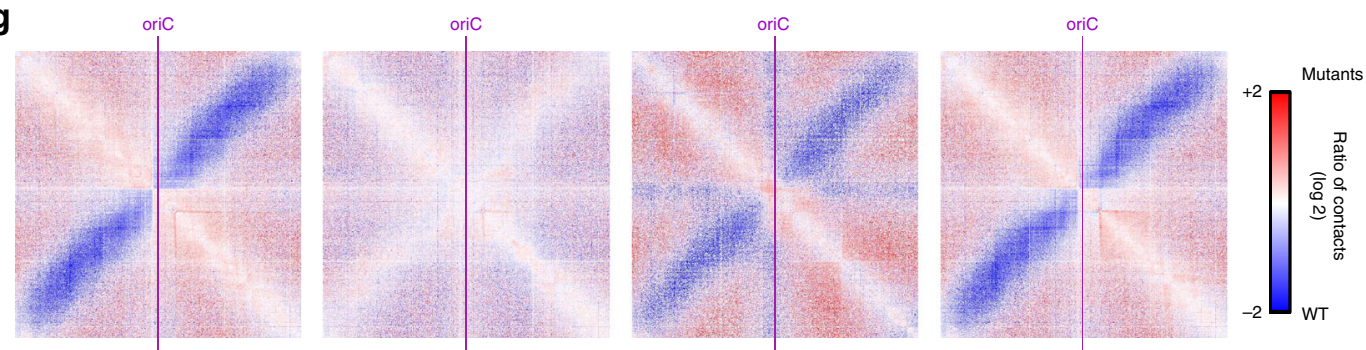

mCherry foci are less frequent in the absence of ParB or parS (Supplementary Fig. 10C). These findings suggest that ParB promote condensin loading onto DNA at oriC-proximal parS sites. In addition, ChIP-seq revealed that SMC concentrates at a $13 \mathrm{~Kb}$ region upstream parS1 (Fig. 4a). SMC enrichment in this region was lost following a partial deletion of this locus and its reinsertion at another genomic position (Supplementary Fig. 10D-F) or following its substitution by a random DNA sequence (Supplementary Fig. 10D, G). Therefore, the accumulation of SMC at the $13 \mathrm{~Kb}$ region in the vicinity of parS sites points at roadblocks that trap SMC rather than specific SMC binding. This hypothesis is further supported by the study of the 
Fig. 3 Functional characterization of two SMC-like complexes in C. glutamicum. a Sections of the C. glutamicum genome map indicating localizations of condensin subunit genes. $\mathbf{b}$ Confirmation of protein-protein interactions via bacterial two-hybrid screen. Interactions were quantified by $\beta$-galactosidase assays in all combinations of hybrid proteins: C/C- (18C/T25), N/C- (18/T25), C/N- (18C/NT25), and N/N- (18/NT25) terminal fusions of adenylate cyclase fragments, ParBRA: ParB mutant R175A (mean \pm SD, $n=3$ ). c Illustration of SMC/ScpAB and MksBEFG subunit interactions based on bacterial twohybrid data; cartoons indicate condensin complex formations. $\mathbf{d}$ Top: dependence of ParB foci numbers on cell length in $C$. glutamicum wild type (WT) and $\Delta$ smc $\Delta m k s B(\Delta \Delta$, CBK011) cells grown in BHI $(n>350)$. Linear regression lines are shown $r(\mathrm{WT})=0.57, r(\Delta \Delta)=0.62$; slopes and intercepts are equal $($ ANCOVA, $F(1,770)=0.059, p=0.808$; ANCOVA, $F(1,771)=0.60, p=0.4391)$. Below: cellular localization of condensin subunits in $C$. glutamicum smc: :smc-mCherry and $m k s B:: m k s B$ - $m$ Cherry cells (CBK012, CBK015). Microscopy images exemplify cellular mCherry fluorescence of SMC (left) and MksB (right); white lines indicate cell outlines. Scale bar, $2 \mu \mathrm{m}$. e Top: SMC and ParB foci numbers positively correlate with cell length in double labeled strain smc: :smc-mCherry parB: :parB-mNeonGreen (CBK013), $r(\operatorname{ParB})=0.74, r(\mathrm{SMC})=0.53(n>350)$. Below: subcellular localization of ParB and SMC is exemplified in representative cells shown in overlays between mNeonGreen and mCherry fluorescence, and in separate channels. Scale bar, $2 \mu \mathrm{m}$. f Normalized contact maps of $\Delta s m c, \Delta m k s B, \Delta p a r B / \Delta s m c$, and $\Delta s m c / \Delta m k s B$ mutants (CDC026, CBK001, CBK002, and CBK004), displayed as in Fig. 1. g Corresponding differential maps between WT and mutant contact maps, indicating the log of the ratio (wild-type norm/mutant norm) are presented as in Fig. 2. Source data are provided as a Source Data file.
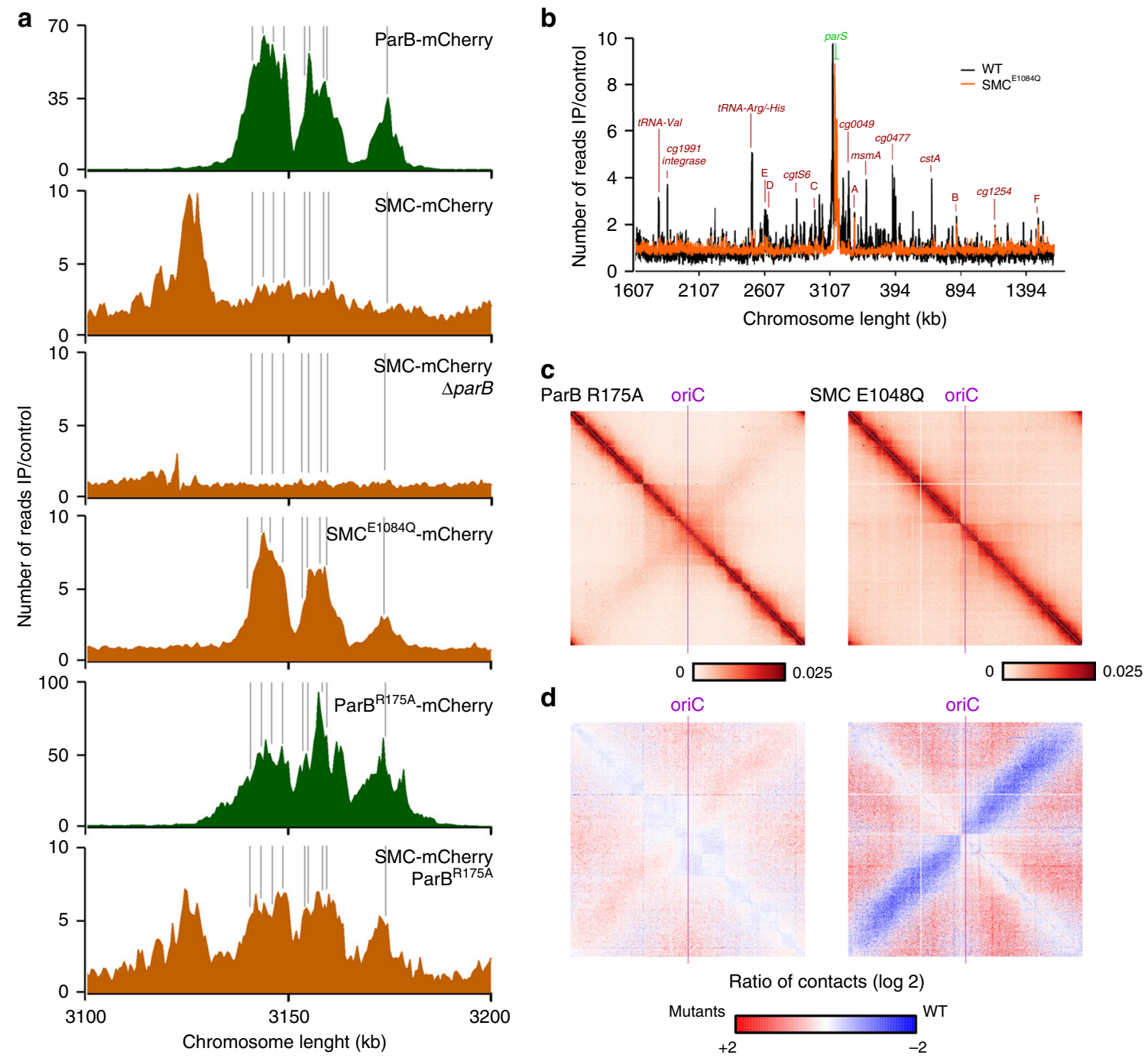

Fig. 4 Chromosomal SMC loading is mediated by ParB at parS sites. a SMC enrichment at parS sites (gray) is ParB-dependent. ChIP-seq of ParB-mCherry (green; CBK006 and CBK047) and SMC-mCherry (orange; CBK012, CBK014, CBK051, and CBK049) in strain backgrounds as indicated. Depicted are chromosomal ranges of 3.1-3.2 Mb, bin size $0.5 \mathrm{~Kb}$. b Whole-genome ChIP-seq data of strains harboring SMC-mCherry wild type (gray, CBK012) or E1084Q mutant (orange, CBK050). SMC enrichment at parS sites and at other loci (red letters), in particular tRNA gene clusters and at rRNA genes (a-f) is illustrated in $0.5 \mathrm{~Kb}$ bins in linear scale along the chromosome with an $x$-axis centered at oriC. c Normalized contact map of mutant strains parB: :parB ${ }^{\mathrm{R} 175 \mathrm{~A}}$ (CBK047) and $\mathbf{d}$ the corresponding differential map indicating the log of the ratio (wild-type norm/mutant norm) as in Fig. 2. 
contact map of wild type cells (Fig. 1c, d and Supplementary Figs. 1 and 11). Indeed, the SMC enrichment region is clearly delimited by a strong border on its left (Supplementary Fig. 1, Directional Index at $100 \mathrm{~Kb}$ resolution and Supplementary Fig. 11 , red dashed line). In the absence of ParB or SMC (Supplementary Fig. 11), the strong border observed in $\mathrm{Hi}-\mathrm{C}$ maps is shifted towards parS sites. Therefore, this border originates from a combination of multiple processes.

SMC is also recruited to parS inserted in ectopic positions, e.g., the $90^{\circ}$ parS-insertion (Supplementary Figs. 5, 6). Indeed, in the absence of SMC (Supplementary Fig. 6), the bow-shape motif is no longer present at the ectopic parS site, demonstrating that chromosomal arm cohesion is SMC-dependent, and that artificial loading of SMC at non-native positions is not sufficient to fold the entire chromosome. We further assayed chromosomal SMCloading sites by making use of a well-characterized SMC ATPhydrolysis mutant $\mathrm{E} 1084 \mathrm{Q}^{32,53-55}$. SMC ${ }^{\mathrm{E} 1084 \mathrm{Q}}$ mutant strongly accumulates at parS sites in C. glutamicum, mimicking a ParBenrichment pattern (Fig. 4a). Decreased ChIP-enrichment signals throughout the rest of the chromosome hint to an impaired SMC migration along the DNA (Fig. 4b). Moreover, the Hi-C contact map of SMC ${ }^{\mathrm{E} 1084 \mathrm{Q}}$ clearly demonstrates that this mutant is no longer able to bridge chromosomal arms (Fig. 4c, d). Conclusively, we confirm specific SMC loading by ParB to an oriCproximal region on the C. glutamicum chromosome.

Interestingly, ChIP analysis of a C. glutamicum $\mathrm{ParB}^{\mathrm{R} 175 \mathrm{~A}}$ mutation, which leads to a loss of dimer-dimer interactions in the corresponding B. subtilis $\mathrm{ParB}^{\mathrm{R} 79 \mathrm{~A}}$ mutation ${ }^{8}$, results in increased SMC binding at $\mathrm{ParB}^{\mathrm{R} 175 \mathrm{~A}}$ propagation zones (Fig. 4a). Changes in in-vitro double-stranded DNA-binding affinities compared with wild-type ParB could not be verified (Supplementary Fig. 12), neither enhanced binding affinity for SMC/ScpAB by bacterial two-hybrid analyses (Fig. 3b). The mutation results in large fractions of DNA-free cells, and growth rates and $\mathrm{ParB}^{\mathrm{R} 175 \mathrm{~A}}$ cluster formation are particularly affected in cells harboring a single parS site (Supplementary Table 1 and Supplementary Fig. 12). ChIP-data indicate broadened and less distinct enrichment signals compared with wild-type ParB in the presence of all or one parS sites (Fig. 4a and Supplementary Figs. 4 and $12 \mathrm{D}, \mathrm{E})$. Therefore, $\mathrm{ParB}^{\mathrm{R} 175 \mathrm{~A}}$ is still capable of building up weak nucleoprotein complexes around parS sites. Hi-C data of the corresponding mutant show the same tendency with a conservation of the overall chromosome architecture with the presence of a secondary diagonal and the conservation of the origin domain folding (Fig. 4c and Supplementary Fig. 11). However, the signal emanating from the secondary diagonal is weak compared with the wild-type one as shown by the ratio matrix (Fig. 4c, d). Consequently, SMC translocation along the DNA appears only partially impaired in this mutant (Fig. 4a and Supplementary Fig. 4). The $\mathrm{ParB}^{\mathrm{R} 175 \mathrm{~A}}$ mutation either locks the translocation ability of SMC/ScpAB by a direct interaction or alterations of $\mathrm{ParB}^{\mathrm{R} 175 \mathrm{~A}}$ nucleoprotein complex properties, namely an incorrect folding of the oriC domain leads to SMC trapping along DNA loops at parS. Altogether, these analyses confirm that the $C$. glutamicum SMC/ScpAB complex is a Bacillus-like condensin that loads and redistributes to distant chromosomal regions via an explicit ParB interaction at parS.

MksB impacts on plasmid maintenance in C. glutamicum. To test whether both C. glutamicum condensins SMC and MksB are redundant in function, we generated mutants lacking the condensin core subunit $\Delta m k s B$ or both $\Delta s m c \Delta m k s B$. Similar to $\Delta s m c$, no growth and morphology phenotypes could be detected for both mutants (Supplementary Fig. 9A, D and Supplementary Table 1). A triple mutation $\Delta$ parB $\Delta s m c \Delta m k s B$ did not aggravate the $\Delta$ parB phenotype, excluding redundancy of condensin functions in chromosome segregation (Supplementary Fig. 9D). Further, oriC-ParB foci numbers (Fig. 3d) and their spatiotemporal localization (Supplementary Fig. 9A) remain largely unaffected upon deletion of $s m c$ and $m k s B$. MksB fluorescence was mainly detected at the cell poles (Fig. 3d), further supporting an interaction with the polar protein DivIVA. To test cellular MksBDivIVA colocalization in more detail, we constructed a dualreporter strain harboring MksB-mCherry in combination with DivIVA-mNeonGreen, which grows and divides in comparasion with the wild type (Fig. 5a, Supplementary Fig. 13A, and Supplementary Table 1). Individual protein fluorescence patterns of MksB and DivIVA are displayed in large-scale demograph analyses (Fig. 5b). Averaged fluorescence profiles along longitudinal cell axes extracted from still microscopy images show colocalization of MksB and DivIVA at cell poles and division septa prior to cytokinesis in long cells (Fig. 5b, c) even if cellular MksB fluorescence intensities are low compared with DivIVA. The relative localization of MksB and DivIVA has also been observed via PALM microscopy. Here we can see that the MksB foci composed of the highest number of localizations typically localize at the poles and are surrounded by DivIVA itself (Supplementary Fig. 13B). Although no quantitative analysis has been performed, the number of visible foci in the imaged cells does not differ with what has been already observed via conventional fluorescence microscopy (Fig. 5a). Moreover, we applied Hi-C to characterize the role of MksB in genome folding in the different mutants (Fig. 3f). In contrast to $s m c$, deletion of $m k s B$ had no effect at large scale on chromosome organization, as shown by the ratio map between the wild type and the mutant (Fig. 3g). Moreover, $\Delta s m c$ and $\Delta s m c \Delta m k s B$ contact maps were nearly identical (Fig. 3f), showing that MksB and SMC are most likely not involved in the same process(es). Finally, we applied the software HiCRep on our various $\mathrm{Hi}-\mathrm{C}$ map, a framework for assessing the reproducibility of $\mathrm{Hi}-\mathrm{C}$ data $^{56}$ (Supplementary Fig. 2A). Strain backgrounds $\Delta p a r B, \Delta p a r S$, and $\Delta p a r B \Delta s m c$ Hi-C maps appear to form a first cluster; $\Delta s m c$ and $\Delta s m c \Delta m k s B$ appear to form a second cluster; finally, wild type, $\Delta p a r S 2-10$, and $\Delta m k s B$ form a third cluster. This result strongly suggests that MksB does not significantly affect chromosome architecture in C. glutamicum. ChIP-seq of MksB failed to detect specific loading sites along the C. glutamicum chromosome (Fig. 5d), supporting the hypothesis that MksB, unlike other bacterial condensins studied so far, plays no direct or indirect role in C. glutamicum chromosome organization. Therefore, we analyzed its impact on the maintenance of extrachromosomal DNA. The MksBEFG complex appears involved in plasmid maintenance, as shown by the qPCR copy number analysis of two low-copy number (pBHK18 and pWK0) and two high-copy number (pJC1 and pEK0) E. coli-C. glutamicum shuttle vectors sized 3.5-6 Kb. High-copy number plasmids derive from cryptic C. glutamicum plasmids ${ }^{57,58}$, whereas replicons of both low-copy number plasmids originate from a plasmid isolated from the closely related Corynebacterium diphteria $e^{59,60}$. In $\Delta m k s B$ mutants, both low-copy number plasmids pBHK18 and pWK0 were enriched 60- and 10-fold compared with wild type, when grown in the absence of selection marker (Fig. 5e). On the contrary, the amount of high-copy number vectors per cell was hardly affected. A $\Delta s m c$ control did not result in a significant increase of plasmid levels compared with wild type (Fig. 5e). We confirmed these findings by plasmid extractions from C. glutamicum cells lacking MksB that yielded exceptionally large quantities of pBHK18 and pWK0, turning them into high-copy number plasmids under these conditions (Fig. 5f). By contrast, amounts of pJC1 and pEK0 did not differ notably compared with control strains. These analyses show a MksB-dependent decrease in plasmid level, specifically of 

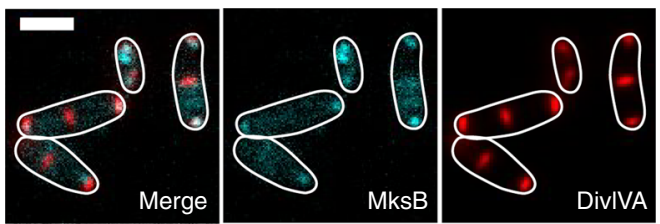

C

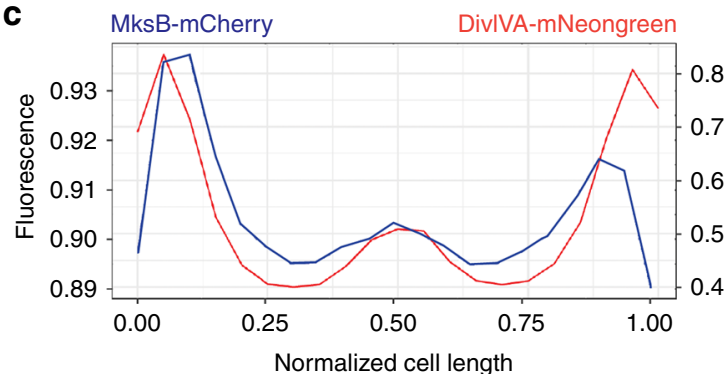

b

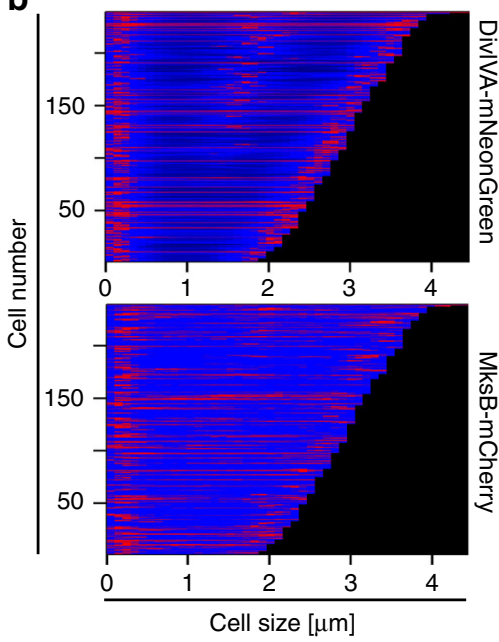

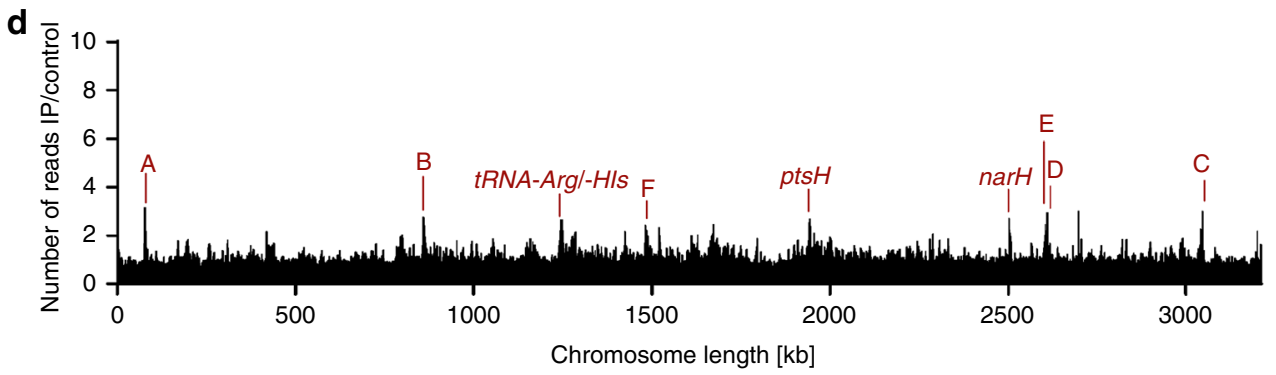
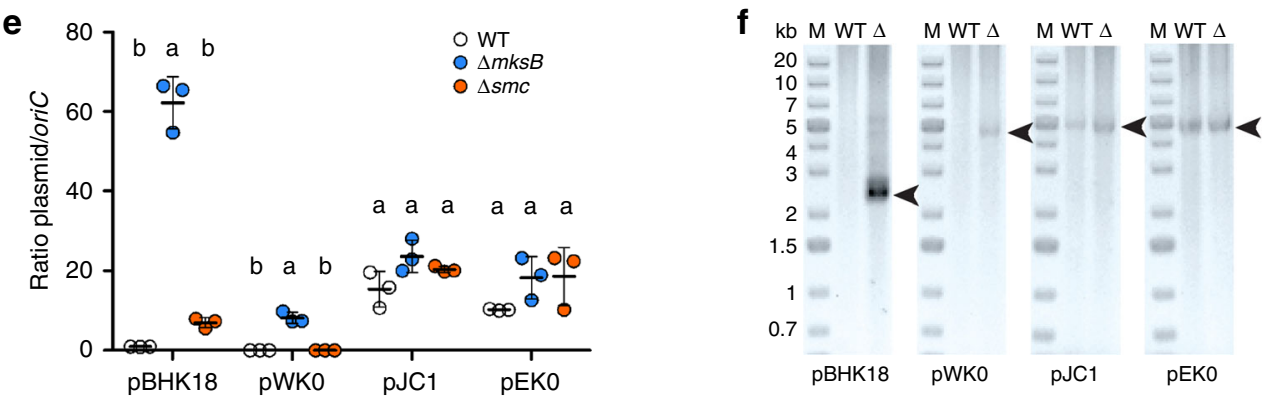

Fig. 5 MksB localizes with DivIVA and impacts on plasmid copy numbers. a Epifluorescence microscopy images of CBK092 cells; MksB-mCherry (cyan) and DivIVA-mNeonGreen fluorescence (red) are shown as overlay and in separate channels; cell outlines are indicated by white lines. Scale bar, $2 \mu \mathrm{m}$. b Demographs show fluorescence profiles of DivIVA and MksB in strain CBK092 along cell axes sorted by length. Fluorescence intensities are illustrated relative to maximal intensity values per cell by a color gradient ranging from dark blue (low intensities) to red (high intensities). c Averaged fluorescence (a.u.) of MksB-mCherry (blue) and DivIVA-mNeonGreen (red) along the longitudinal cell axis of CBK092 cells ( $n>200$ cells). Fluorescence values of profiles were normalized in length and fluorescence intensity per cell. The resulting values were then binned (bin $=0.05 \mu \mathrm{m})$. $\mathbf{d}$ Anti-mCherry-ChIP-seq analysis of $m k s B:: m k s B-m C h e r r y$ strain CBK015 as described in Supplementary Fig. 4. e Plasmid copy numbers of low-copy (pBHK18 and pWK0) and highcopy number vectors ( $\mathrm{pJC} 1$ and $\mathrm{pEC} 0$ ) relative to oriC numbers per cell, assayed by qPCR. Ratios were compared between $C$. glutamicum wild type, $\Delta m k s B$, and $\Delta s m c$ mutant cells grown in $\mathrm{BHI}$ medium without addition of plasmid selection antibiotic after overnight pre-incubation with antibiotic (mean $\pm \mathrm{SD}$, $n=3)$. One-way ANOVAs yielded significant variations among strains harboring $\mathrm{pBHK18}(\mathrm{F}(2,6)=233.3, p=2.05 \mathrm{e}-06)$ and $\mathrm{pWKO}(\mathrm{F}(2,6)=98.66$, $p=2.57 \mathrm{e}-05)$, but not among strains harboring $\mathrm{pEKO}(\mathrm{F}(2,6)=2.496, p=0.163)$ and $\mathrm{pJC1}$ plasmids $(\mathrm{F}(2,6)=51.75, p=0.0739)$. Letters indicate significant differences between data sets determined by post-hoc Bonferroni analysis at $p<.05$. f Plasmids named in a were extracted from $C$. glutamicum wild type and $m k s B$ deletion strains grown in $\mathrm{BHI}$ medium including selection antibiotic, visualization of extracted DNA on $1 \%$ agarose gels (corresponds to yield from $\sim 1 \times 10^{9}$ cells each). Arrows indicate size of plasmid DNA. Source data are provided as a Source Data file.

low-copy number plasmids. Notably, we observed emerging susceptibility of cells towards the pBHK18 selection antibiotic in the absence of MksB when testing its stability in plating assays and, therefore, cannot exclude side effects of MksB on the expression of antibiotic resistance. Subcellular MksB-mCherry localization was further assessed in the absence and presence of pEK0 or pWK0. The presence of plasmids does not have an impact on cell growth and morphology (Supplementary Fig. 13C) and wild-type-like MksB foci numbers per cell were detected (Supplementary Fig. 13D). Cellular MksB fluorescence profiles were further extracted (Supplementary Fig. 13E) and sorted by cell length in demographs showing MksB localization at cell poles and frequently at midcell prior to cytokinesis for all conditions (Supplementary Fig. 13E, F). However, polar MksB fluorescence tends to be more defined in cell populations harboring plasmids (Supplementary Fig. 13E, F). 
Altogether, our data show that the two condensins in $C$. glutamicum evolved very different functions: whereas SMC/ $\mathrm{Scp} A B$ act with ParB to promote replichore pairing and origin domain organization, MksBEFG does not organize chromosome architecture and seems involved in plasmid maintenance through a mechanism that remains to be characterized.

\section{Discussion}

Condensins are widely conserved enzyme machineries, which have been implicated in chromosome organization of pro- and eukaryotes $^{61}$. For long, it was considered that bacterial genomes encode one condensin complex that would either be of the Smc/ ScpAB type as found in B. subtilis and C. crescentus or the MukBEF complex encoded in E. coli and related proteobacteria ${ }^{23}$. However, recent reports suggested the existence of two or even multiple condensin systems in a single species ${ }^{40}$. Yet, the underlying mechanisms and the precise function of these two condensin systems remained largely untested. We report here that the Gram-positive actinobacterium C. glutamicum also contains SMC/ScpAB and the Muk-like MksBEFG complexes. We set out to address the individual functions of the two condensin systems. Surprisingly our data provide clear evidence that the class of MksBEFG proteins do not work as chromosomal interactors; thus, the function of bacterial condensins in promoting DNA segregation to daughter cells is not generally conserved. A recent bioinformatics study predicted a role for MksBEFG complexes (termed Wadjet system) in plasmid-related defense, where heterologous complex expression conveyed protection against the uptake of a high-copy number plasmid ${ }^{62}$. However, function of MksBEFG in its native host had not been addressed before. We could show that the Mks system is indeed involved in the control of plasmid copy numbers, and that there is no involvement of this system in chromosome organization. As low-copy number vectors used in this study harbor replicons of a related Corynebacterium genus, the impact of MksBEFG on plasmids may be based on adaptations of plasmid-specific characteristics to the host species, such as the structural organization of the replication origin or replication-associated proteins ${ }^{63-65}$. Our findings share a fascinating similarity to specific eukaryotic condensing homologs such as Rad50, being the closest eukaryotic relative to $\mathrm{MukB} / \mathrm{MksB}^{23,40}$. It was recently shown that Rad50-CARD9 complexes sense foreign cytoplasmic DNA in mammalian cells acting in innate immune responses against viral $\mathrm{DNA}^{66}$. In addition, the more distantly related eukaryotic SMC5/6 complex had been shown to act in a defense mechanism against circular hepatitis B virus DNA, resembling the specific effect of prokaryotic MksBEFG on plasmids ${ }^{67}$. Together, our data lend support to the notion that condensins' function in innate immunity is an ancient mechanism. However, notably, we provide evidence that the MksBEFG complex is the only known condensin amongst pro- and eukaryotes to date that exclusively impacts on nonchromosomal DNA. For MksBEF systems, it has been proposed that a fourth subunit, MksG, is important for function in plasmid maintenance ${ }^{62}$. We could verify that MksG is part of the MksBEF complex of C. glutamicum. This assumption is in line with divergent functions observed between C. glutamicum MksBEFG and the structurally related $P$. aeruginosa MksBEF complex that is assumed to act in chromosome organization due to a synthetic DNA-segregation phenotype in combination with $\mathrm{SMC} / \mathrm{ScpAB}^{40}$. The direct interaction of a Mks complex with a polar scaffold protein such as the C. glutamicum DivIVA has not been described before. A challenging question for the future will be to determine the detailed mechanism of MksBEFG in plasmid defense and the putative role of the DivIVA-Mks interaction in this process.
We further describe here that $\mathrm{SMC/ScpAB}$ is indeed the major factor of replichore cohesion and chromosome organization in C. glutamicum. Like in B. subtilis, SMC is preferentially loaded onto the chromosome by a ParB/parS loading complex before spreading to the entire chromosome. The mild DNA-partitioning defects of a $s m c$ deletion in combination with a ParB-eYFP modification (Supplementary Table 1) strongly suggest a supportive role of SMC/ScpAB in the process of nucleoid separation, yet the $s m c$ phenotype appears to be entirely compensated by ParB. Therefore, our data demonstrate that the conserved role for SMC in chromosome organization ${ }^{10,20-22,35,36}$ is also maintained in C. glutamicum. Moreover, bacterial two-hybrid analyses of $\mathrm{SMC} / \mathrm{ScpAB}$ subunits evidence a self-interaction of C. glutamicum kleisin ScpA (Fig. 3), which has not been described in other organisms before. Based on this result, we speculate that C. glutamicum SMC/ScpAB might form dimers via kleisin subunits similar to $E$. coli MukBEF complex ${ }^{68,69}$. These data point to a handcuffing model, where two SMC/ScpAB complexes are physically coupled together and translocate in pairs along the chromosome, similar as suggested for B. subtilis ${ }^{31}$. We further describe a new phenotype for a $\mathrm{ParB}^{\mathrm{R} 175 \mathrm{~A}}$ point mutation in $C$. glutamicum that decreases SMC recruitment or blocks SMC release from its loading site. Building on this, we observe a weak interaction signal of ParB ${ }^{\mathrm{R} 175 \mathrm{~A}}$ with ScpB in bacterial two-hybrid analyses. Alternatively, SMC/ScpAB remains indirectly entrapped in higher-order ParB ${ }^{R 175 A}$ nucleocomplexes, which possess altered DNA-folding properties. In either case, this mutant underlines the crosstalk between SMC/ScpAB and ParB nucleoprotein complexes in bacterial nucleoid organization.

Analysis of ParB complexes using two-dimensional (2D) PALM reveals ParB-dense regions within clusters that correlate to the number of ParB-enrichment zones along adjacent parS sites. In line with a current study on ParB cluster-assembly in $V$. cholerae $^{70}$, we suggest that these subclusters derive from independent nucleation and caging events, which merge into one ParB-macrocomplex per oriC in C. glutamicum. Presence of a single parS site leads to formation of almost globular ParB densities. Using Hi-C approaches, we further show that parS sites and ParB are major factors of chromosome folding in C. glutamicum as previously shown in other organisms ${ }^{10,21,22,36}$. C. glutamicum chromosome adopts a global folding with a strong cohesion between the two chromosomal arms as expected from a bacterium harboring a longitudinal chromosomal organization similar to B. subtilis and, to a lesser extent, C. crescentus (Fig. 1). Our analysis also suggests the existence of a chromosomal domain at parS sites in C. glutamicum as previously observed in B. subtilis, but with important differences: parS sites in C. glutamicum are only found on one side of the oriC locus and appeared to be at the edge of the nucleoid structure as observed in C. crescentus. A hairpin structure as it was observed in B. subtilis is absent in C. glutamicum ${ }^{10}$. Contact maps of a strain with an ectopic parS site feature a bow-shaped structure reflecting an asymmetry in arm interaction, which has been shown before in B. subtilis and C. crescentus $^{21,22}$. Zipping of the chromosome is not complete and the ectopic parS site does not reorient the entire chromosome. Therefore, additional factors are involved in chromosome localization that supplement polar ParB-parS binding to DivIVA.

Importantly, we describe $\mathrm{ParB} /$ parS-dependent DNA contacts of the parS region with the entire nucleoid, indicating that oriC segregation occurs across the entire nucleoid. This is in accord with the ori-ter configuration of the nucleoid in C. glutamicum. Different from C. glutamicum, B. subtilis SMC is required for segregation signals that do not spread along the whole chromosomal length ${ }^{21}$. Based on our data, we propose the following model shown in Supplementary Fig. 14: organisms with polarly localized oriCs and a longitudinal chromosome organization rely 
on ParAB for oriC segregation, as they can use the DNA scaffold as a track. By contrast, species with a central replication factory cannot efficiently use ParAB. B. subtilis is an exception, since here a longitudinal chromosome orientation is present during sporulation and, hence, $\operatorname{par} A B(s p o 0 \mathrm{~J} / \mathrm{soj})$ phenotypes are only obvious during spore formation. Segregation of otherwise transversally arranged $B$. subtilis chromosomes during vegetative growth rely on an initial SMC-driven oriC segregation along a limited fraction of the nucleoid instead ${ }^{21}$. Consequently, SMC/ ScpAB-mediated replichore cohesion is likely dispensable for oriC segregation in bacteria with a strict longitudinal chromosome arrangement that allows for efficient ParABS-driven chromosome partitioning.

\section{Methods}

Bacterial strains, plasmids, and oligonucleotides. Primers, plasmids, and strains used in this study are listed in Supplementary Data 1 and 2.

For protein-protein interaction screens, genes of interest were amplified via PCR, digested with respective enzymes, and ligated into bacterial two-hybrid vectors $^{71}$. E. coli DH5a were utilized for plasmid cloning. Genes divIVA and parB/parB $B^{\mathrm{R} 175 \mathrm{~A}}$ were amplified using primer pairs DivIVA-XbaI-F/DivIVABamHI-R and ParB-XbaI-F/ParB-BamHI-R from genomic DNA or pK19mobsacBParBR175A, and resulting fragments were digested with XbaI/BamHI. For amplification of $s c p A, s c p B, m k s E, m k s F$, and $m k s G$, primer pairs ScpA-XbaI-F/ ScpA-XmaI-R, ScpB-XbaI-F/ScpB-XmaI-R, MksE-XbaI-F/MksE-XmaI-R, MksFXbaI-F/MksF-XmaI-R, and MksG-XbaI-F/MksG-XmaI-R were utilized, followed by restriction digests with XmaI/XbaI. Primer pairs SMC-XbaI-F/SMC-KpnI-R and MksB-XmaI-F/MksB-KpnI-R were used for PCR amplification of genes smc and $m k s B$, which were subsequently digested with $\mathrm{XbaI} / \mathrm{KpnI}$ or $\mathrm{XmaI} / \mathrm{KpnI}$. To increase the distance of $\mathrm{XmaI}$ and $\mathrm{KpnI}$ restriction sites, a short sequence was inserted in between these sites by overhang PCRs using pUT18C-mcs-HindIII-F, pUT18-mcs-PvuII-F, pKNT25-mcs-NheI-F, or pKT25-mcs-HindIII-F in combination with pUT18(C)/pK(N)T25-mcs-KpnI-R for plasmids pUT18C, pUT18, pKT25, and pKNT25, respectively. Resulting fragments and corresponding vectors were digested with HindIII/KpnI, PvuII/KpnI, or NheI/KpnI and subsequently ligated, resulting in plasmids pUT18 mcs, pUT18C mcs, pKNT25_mcs, and pKT25_mcs. All digested gene fragments mentioned above were ligated into pUT18, pUT18C, pKNT25, and pKT25 or pUT18_mcs, pUT18C_mcs, pKNT25_mcs, and pKT25_mcs, respectively.

Derivatives of the suicide integration vector pK19mobsacB were used for clean allelic replacements in C. glutamicum, containing the modified genomic region of interest including its $500 \mathrm{bp}$ up- and downstream homologous flanking sequences. Plasmid cloning was performed using E. coli $\mathrm{DH} 5 \mathrm{a}$.

To construct pK19mobsacB- $\Delta$ smc $500 \mathrm{bp}$ upstream and downstream of $s m c$ were PCR amplified using primer pairs $\Delta$ smc-BamHI-up-F/ $\Delta$ smc-up-R and $\Delta$ smc$\mathrm{D}-\mathrm{F} / \Delta$ smc-EcoRI-D-R, respectively. Both fragments served as templates in an overhang PCR, yielding a 1000 bp fragment, which was digested with BamHI and EcoRI and subsequently ligated into pK19mobsacB. pK19mobsacB- $\triangle$ SMCload was constructed accordingly, using primer pairs $\triangle$ SMCload-HindIII-up-F/ $\Delta$ SMCloadup-R and $\Delta$ SMCload-D-F/ $\Delta$ SMCload-SalI-D-R, and HindIII in combination with SalI for restriction digest. For construction of pK19mobsacB- $\Delta$ mksB up-/and downstream regions of $m k s B$ were PCR amplified using primers $\Delta$ mksB-HindIIIup-F/ $\Delta$ mksB-PstI-up-R and $\Delta$ mksB-PstI-D-F/ $\Delta$ mksB-XbaI-D-R. Resulting 500 bp fragments were digested with HindII/ PstI and PstI/ XbaI and consecutively ligated into pK19mobsacB.

Fluorescent C-terminal fusions of ParB protein with PAmCherry or mNeongreen were obtained by utilizing plasmids pK19mobsacB-parBmNeonGreen and pK19mobsacB-parB-PAmCherry. To this end, the eYFP sequence of plasmid pK19mobsacB-parB-eYFP ${ }^{41}$ was replaced by respective fluorophore sequences, which were amplified via PCR using PAmCherry-SalI-F/ PAmCherry-XbaI-R primers and digested with SalI and XbaI.

For fluorescent versions of SMC and MksB proteins plasmids pK19mobsacBsmc-mCherry, pK19mobsacB-mksB-mCherry and pK19mobsacB-mksB-

PAmCherry were constructed. At first, $500 \mathrm{bp}$ regions up- and downstream of the $3^{\prime}$-end of $s m c$ or $m k s B$ were amplified using primer pairs SMC-HindIII-up-F/SMCSphI-up-R and SMC-BamHI-D-F/SMC-EcoRI-D-R or MksB-HindIII-up-F/MksBSph-up-R and MksB-BamHI-D-F/MksB-EcoRI-D-R. Fluorophore sequences were amplified with primers PAmCherry-SalI-F/mCherry-BamHI-R for SMC-mCherry and MksB-PAmCherry fusion or with primers PAmCherry-SalI-F/mCherry-XbaI$\mathrm{R}$ for the MksB-mCherry fusion construct. Up- and downstream fragments were digested via HindIII/SphI and BamHI/EcoRI, whereas enzymes SalI/BamHI or SalI/XbaI were utilized for restriction digest of fluorophore sequences fused to $s m c$ or $m k s B$, respectively. Fragments were subsequently ligated into the pK19mobsacB plasmid, starting with the corresponding downstream region, followed by the fluorophore sequence and finally the upstream region.

To place part of a putative SMC binding site upstream of the parS cluster into an intergenic region $3^{\prime}$ of cg0177 (Supplementary Fig. 10), genomic sequences
$500 \mathrm{bp}$ up- and downstream of the insertion site were amplified using primer pairs cg0177-HindIII-up-F/cg0177-SalI-up-R and cg0177-XmaI-D-F/cg0177-EcoRI-D$\mathrm{R}$; part of the genomic SMC binding site $(1.1 \mathrm{~Kb})$ was amplified using primers SMCload-SalI-F and SMCload-XmaI-R. Resulting fragments were digested with HindII/SalI, SalI/XmaI, and XmaI/EcoRI, and consecutively ligated into pK19mobsacB, obtaining the plasmid pK19mobsacB-SMCload-cg0177.

Plasmid pK19mobsacB-SMCload-r was constructed for the partial replacement of the SMC binding site $(1.1 \mathrm{~Kb})$ with a $B$. subtilis genomic region of identical size. For amplification of up- and downstream $500 \mathrm{bp}$ regions, primer pairs $\triangle S M C l o a d$ HindIII-up-F/SMCload-SphI-up-R and SMCload-PstI-D-F/ $\Delta$ SMCload-SalI-D-R were utilized, whereas the replacement sequence was amplified from $B$. subtilis genomic DNA via SMCloadr-SphI-F/SMCloadr-PstI-R. After digestion with enzymes HindIII/SphI, PstI/SalI, or SphI/PstI, fragments were successively ligated into pK19mobsacB

Further, all parS sites were mutated comprising new XmaI or SalI restriction sites (see Supplementary Fig. 2). For mutation of parS1 primer pairs, parS1mutHindIII-up-F/parS1mut-XmaI-up-R and parS1mut-XmaI-D-F/parS1mut-EcoRI$\mathrm{D}-\mathrm{R}$ were utilized to mutate parS1 and to amplify sequences $500 \mathrm{bp}$ up- and downstream of parS1. Restriction digest was performed with both fragments using HindIII/XmaI or XmaI/EcoRI, respectively. Subsequent ligation into pK19mobsacB yielded plasmid pK19mobsacB-parS1mut. To mutate parS2, parS3, parS4, parS7, and parS8, plasmid construction was performed in the same way using primers parS2mut-HindIII-up-F/parS2mut-XmaI-up-R and parS2mut-XmaI-D-F/ parS2mut-EcoRI-D-R, parS3mut-HindIII-up-F/parS3mut-XmaI-up-R and parS3mut-XmaI-D-F/parS3mut-EcoRI-D-R, parS4mut-HindIII-up-F/parS4mutXmaI-up-R and parS4mut-XmaI-D-F/parS4mut-EcoRI-D-R, parS7mut-HindIII up-F/parS7mut-XmaI-up-R and parS7mut-XmaI-D-F/parS7mut-EcoRI-D-R, or parS8mut-HindIII-up-F/parS8mut-XmaI-up-R and parS8mut-XmaI-D-F/ parS8mut-EcoRI-D-R for amplification of fragments up- and downstream of the respective parS site. Matching fragments were each digested and ligated into pK19mobsacB, as exemplified for pK19mobsacB-parS1mut construction, resulting in plasmids pK19mobsacB-parS2mut, pK19mobsacB-parS3mut, pK19mobsacBparS4mut, pK19mobsacB-parS7mut, and pK19mobsacB-parS8mut.

As parS5 and parS6, as well as parS9 and parS10, are localized in close proximity on the genome ( $<100 \mathrm{bp}$ distance), their deletions were accomplished using in each case one plasmid for both parS sites. For construction of pK19mobsacB-parS5_6mut genomic region upstream of parS5, downstream of parS6, and in between, both sides were PCR amplified using parS5mut-HindIII-up-F/parS5mut-SalI-up-R, parS6mut-XmaI-D-F/parS6mut-EcoRI-D-R, and parS5mut-SalI-D-F/parS6mutXmaI-up-R, and fragments were digested with HindIII/SalI, XmaI/EcoRI, or SalI/ XmaI, respectively, and ligated into $\mathrm{pK} 19 \mathrm{mobsacB}$. Construction of pK19mobsacBparS9_10mut was performed accordingly, using primer pairs parS9mut-HindIII-up F/parS9mut-SalI-up-R, parS10mut-XmaI-D-F/parS10mut-EcoRI-D-R, and parS9mut-SalI-D-F/parS10mut-XmaI-up-R for fragment amplification.

Insertion of parS $3^{\prime}$ of $\operatorname{cg} 0108, \operatorname{cg} 0904$, and $\operatorname{cg} 2563\left(9.5^{\circ}, 90^{\circ}\right.$, and $270^{\circ}$ chromosomal positions) were achieved via plasmids pK19mobsacB-parS-cg0108, pK19mobsacB-parS-cg0904, and pK19mobsacB-parS-cg02563. Primers containing parS sites were used to amplify regions $500 \mathrm{bp}$ up- and downstream of the corresponding parS insertion site, namely parS-cg0108-SalI-up-F/parS-cg0108-up$\mathrm{R}$ and parS-cg0108-D-F/parS-cg0108-XmaI-D-R, parS-cg0904-HindIII-up-F/parScg0904-up-R and parS-cg0904-D-F/parS-cg0904-NheI-D-R, or parS-cg2563HindIII-up-F/parS-cg2563-up-R and parS-cg2563-D-F/parS-cg2563-NheI-D-R, respectively. Each fragment pair served as template in an overhang PCR, yielding $1000 \mathrm{bp}$ sequences with central parS sites. After restriction digest with SalI/XmaI or HindIII/NheI, each fragment was ligated into pK19mobsacB. Plasmid pK19mobsacB-parS- $\Delta$ int for parS insertion at ter $C$ was constructed in the same way, however by replacing an entire gene (cg1752, $180^{\circ}$ chromosomal position). Regions $500 \mathrm{bp} \mathrm{N}$ - and C-terminally of $c g 1752$ were amplified using parS- $\Delta$ intHindIII-up-F/parS- $\Delta$ int-up-R and parS- $\Delta$ int-D-F/parS- $\Delta$ int-NheI-D-R.

For construction of pK19mobsacB-parBR175A, primer pairs ParB-N-terHindIII-F/ParB-R175A-R and ParB-R175A-F/ParB-C-ter-SalI-R were used to amplify the $\mathrm{N}$ - and $\mathrm{C}$-terminal parts of parB surrounding the coding region of $\mathrm{ParB}^{\mathrm{R} 175}$. Primers introduce point mutations into this codon and into a neighboring SacI restriction site, resulting in fragments of $528 \mathrm{bp}$ and $625 \mathrm{bp}$ length. Overhang PCR yielded a full parB sequence that was cut with HindIII/Sall and ligated into pK19mobsacB. pK19mobsacB-smcE1084Q was obtained in an analogous manner. Amplification of $500 \mathrm{bp}$ genomic regions surrounding codon SMCE1084 were performed using primer pairs E1084Q-HindIII-up-F/E1084Q-up-R and E1084Q-D-F/E1084Q-BamHI-D-R, which further yield in an E1084Q mutation and an additional XbaI restriction site $3^{\prime}$ of the codon sequence.

His-tagged versions of $\mathrm{ParB}$ and $\mathrm{ParB}^{\mathrm{R} 175 \mathrm{~A}}$ were generated by applying PCR (ParB-NdeI-F/ParB-XhoI-R) following a restriction digest (NdeI/XhoI) of the respective DNA fragment and ligation into $\mathrm{pET}-16 \mathrm{~b}$ expression vector yielding pET-16b-ParB and pET-16b-ParBR175A

For construction of the E. coli-C. glutamicum shuttle expression vector pEKEx2-mCherry the mCherry sequence was amplified via PCR using mCherrySacI-F/ mCherry-EcoRI-R, digested with corresponding restriction enzymes, and ligated into the empty pEKEx2.

Vectors were transformed via electroporation into C. glutamicum cells ${ }^{72}$. Genomic integration of $\mathrm{pK} 19$ mobsacB plasmids were selected on kanamycin, whereas the second crossover event was confirmed by growth on $10 \%$ sucrose. 
Screening of allelic replacements in C. glutamicum $\Delta s m c, \Delta m k s B$, and $\Delta p a r B$ was performed by colony PCR using primer pairs $\Delta$ smc-seq-700up-F/ $\Delta$ smc-seq-700DR, $\Delta$ mksB-seq-700up-F/AmksB-seq-700D-R, and ParB-seq-800up-F/ParB-seq800D-R. Fluorescent fusions of ParB, SMC, and MksB were confirmed via primer pairs ParB-N-ter-SalI-F/ParB-seq-800D-R, SMC-seq-1589bp-F/Asmc-seq-700D-R, and MksB-seq-1595bp-F/ $\Delta$ mksB-seq-700D-R, respectively. Insertions of the partial smc loading site in an intergenic region $3^{\prime}$ of $c g 0177$ were screened using primer pairs cg0177-seq-700up-F/cg0177-seq-700D-R. To identify genomic parS mutations, respective regions were amplified using upstream-forward and downstream-reverse primers as used for plasmid construction and digested with either XmaI or SalI. Sequencing of parS loci was performed for further verification. For verification of parS insertions $3^{\prime}$ of $c g 0108$, cg0904, and $c g 2563$ or for replacement of $c g 1752$ by parS genomic loci were amplified with primers cg0108seq-400up-F/cg0108-seq-200D-R, cg0904-seq-100up-F/cg0904-seq-100D-R, cg2563-seq-200up-F/cg2563-seq-300D-R, or $\Delta$ int-seq-700up-F/Dint-seq-700D-R, respectively, followed by a control restriction digest using PmlI. Screening for parB $B^{R 175 A}$ was performed by amplification of parB including $800 \mathrm{bp}$ up- and downstream regions via primers ParB-seq-800up-F/ParB-seq-800D-R. A control digest was conducted with the resulting fragment using SacI. Integration of the point mutation $s \mathrm{c}^{\mathrm{E} 1084 \mathrm{Q}}$ was verified by amplification of the respective genomic region (E1084Q-HindIII-up-F/mCherry-EcoRI-R), followed by restriction digests using XbaI.

Assembly strategies of multiple consecutive allelic replacements are explained hereafter. C. glutamicum strains CBK002, CBK004, and CBK010 were obtained via transformation of pK19mobsacB- $\Delta$ parB, pK19mobsacB- $\Delta$ mksB, or pK19mobsacBparB-eYFP into strain CDC026 lacking $s m c$ and strain CBK003 $(\Delta m k s B \Delta p a r B)$ was constructed using the genetic background of CBK001 $(\Delta m k s B)$. Further, CBK004 served as parent strain for construction of CBK005 and CBK011 harboring additional mutations $\triangle$ parB and parB: :parB-eYFP, respectively. The dual-reporter strain CBK013, expressing ParB-mNeonGreen in combination with SMC-mCherry, was constructed via transformations of pK19mobsacB-smc-mCherry into CBK008; strain CBK014 derives from CBK012 transformed with pK19mobsacB- $\Delta$ parB. The complete loss of parS sites in strain CBK024 was accomplished via successive allelic replacements of parS by mutated sequences: the mutation of parS2 (CBK017) followed the mutation of parS3 (CBK016); thereupon, parS4 (CBK018) was mutated followed by parS5 and parS6 (CBK019). Next, parS7 (CBK020) mutation, parS8 mutation (CBK021), parS9 mutation (CBK022), parS10 mutation (CBK023), and parS1 mutation (CBK024) were accomplished consecutively. CBK090 is a derivative of CBK022. CBK025, CBK027, and CBK029 derive from strain CBK023, which was transformed with $\mathrm{pK} 19 \mathrm{mobsacB}$ plasmids coding for parB-eYFP, parB-mCherry2, and parB-PAmCherry, respectively. Accordingly, stains CBK026, CBK28, CBK032, and $\mathrm{CBK} 087$ are $\mathrm{CBK} 024$ derivatives harboring either endogenous parB-eYFP, parB-mCherry2, smc-mCherry, or parB-PAmCherry, whereas strains CBK030 and CBK031 obtained from CBK022 via transformation of pK19mobsacB-parBmCherry2 or pK19mobsacB-parB-PAmCherry. CBK091 was obtained by transformation of CBK090 with pK19mobsacB-parB-mCherry2. CBK033 and CBK035 were generated by transformation of strain CBK012 expressing SMCmCherry with plasmids pK19mobsacB- $\Delta$ SMCload or pK19mobsacB-SMCload-r; a further transformation of CBK033 with pK19mobsacB-SMCload-cg0177 yielded CBK034. To introduce parS sites at different regions within the C. glutamicum genome CBK024 served as parental strain: parS-insertions at chromosomal $9.5^{\circ}, 90^{\circ}$, $270^{\circ}$, and $180^{\circ}$ positions were achieved via transformation of either $\mathrm{pK} 19 \mathrm{mobsacB}-$ parS-cg0108 (CBK036), pK19mobsacB-parS-cg0904 (CBK037), pK19mobsacBparS-cg2563 (CBK038), or pK19mobsacB-parS- $\Delta$ int (CBK039). Additional allelic replacements of parB or $s m c$ with fluorophore-coupled versions parB-eYFP or parB$m$ Cherry2 and smc-mCherry in the above-named strains resulted in CBK040CBK045. Second, parS-insertion in CBK037 was combined with a $s m c$ deletion by transformation of pK19mobsacB- $\Delta$ smc yielding CBK046. Lastly, strains CBK047-CBK051, which express mutant ParB ${ }^{\mathrm{R} 175 \mathrm{~A}}$ or SMC $\mathrm{C}^{\mathrm{E} 1084 \mathrm{Q}}$ proteins derive from CBK006, CBK027, and CBK012 transformed with plasmid pK19mobsacBparBR175A or pK19mobsacB-smcE1084Q, respectively. GGCB1C8 was used for subsequent construction of strains CBK092 and CBK093 by transformation with either pK19mobsacB-mksB-mCherry or pK19mobsacB-mksB-PAmCherry.

Plasmid extraction from C. glutamicum cells. C. glutamicum cells were grown in $10 \mathrm{ml} \mathrm{BHI} \mathrm{medium} \mathrm{to} \mathrm{exponential} \mathrm{growth} \mathrm{phases} \mathrm{in} \mathrm{presence} \mathrm{of} \mathrm{selection} \mathrm{anti-}$ biotic, following incubation with $20 \mathrm{mg} / \mathrm{ml}$ lysozyme in P1 buffer (NucleoSpin ${ }^{\oplus}$ Plasmid Kit, Macherey-Nagel) overnight at $30^{\circ} \mathrm{C}$. Subsequently, plasmids were extracted by using the plasmid kit according to manufacturer's instruction.

Growth conditions and media. E. coli cells were grown at $37^{\circ} \mathrm{C}$ in Lysogeny Broth (LB) medium supplemented with $50 \mu \mathrm{g} / \mathrm{ml}$ kanamycin when appropriate. Growth experiments of C. glutamicum cells were performed using brain heart infusion medium (BHI, Oxoid ${ }^{\mathrm{TM}}$ ) or CGXII medium ${ }^{73}$ supplemented with $4 \%$ glucose or $120 \mathrm{mM}$ acetate at $30^{\circ} \mathrm{C}$. Cells were always preinoculated in BHI overnight; for growth in minimal media cells were first inoculated in BHI and rediluted in the corresponding growth media overnight for pre-cultivation. Finally, cell cultures were adjusted to an $\mathrm{OD}_{600}$ of 0.5 for $\mathrm{BHI}$ and to an $\mathrm{OD}_{600}$ of 1 for growth in CGXII medium. Kanamycin $(25 \mu \mathrm{g} / \mathrm{ml})$ was added where applicable.

\section{Protein identification via immunoprecipitation and mass spectrometry.}

Immunoprecipitation of SMC and MksB interaction partners was performed with strains CBK012 and CBK015, further including strain CBK052 as negative control. Lysate of exponentially grown cells was used for immunoprecipitation via magnetic RFP-Trap ${ }^{\circledR}$ agarose beads. For proteomic analysis samples were further processed and analyzed by liquid chromatography tandem mass spectrometry (LC-MS/MS) to identify and quantify proteins in all samples.

For immunoprecipitation of interacting proteins, strains CBK012, CBK015, and CBK052 were cultivated in BHI medium using culture flasks pretreated with $0.5 \%$ sodium hypochlorite. CBK052 was induced at OD600 1 with $0.5 \mathrm{mM}$ isopropyl $\beta$-D-1-thiogalactopyranoside (IPTG). Exponentially growing cells $(\mathrm{OD} 600=3$, $10 \mathrm{ml}$ ) were collected, washed once in $10 \mathrm{ml}$ washing buffer (Tris- $\mathrm{HCl} \mathrm{pH} 7.5$ $10 \mathrm{mM} ; \mathrm{NaCl} 150 \mathrm{mM}$; EDTA $0.5 \mathrm{mM}$ ) and resuspended in $1.5 \mathrm{ml}$ washing buffer supplemented with $1 \mathrm{mM}$ phenylmethylsulfonyl fluoride in EtOH. All following steps were performed at $4^{\circ} \mathrm{C}$. After cell disruption via FastPrep ${ }^{\oplus}$-24 (MP Biomedicals) at $10 \times 6.5 \mathrm{~m} / \mathrm{s}, 30 \mathrm{~s}$ cell debris was removed by centrifugation at $18,000 \times g$. Immunoprecipitation was performed with $25 \mu$ l magnetic RFP-Trap ${ }^{\oplus}$ agarose beads (Chromotek) incubated in $1 \mathrm{ml}$ Lysate for $1 \mathrm{~h}$. Thereupon, beads were washed three times in washing buffer and again washed three times in $100 \mathrm{mM}$ ammonium bicarbonate prior to storage at $-20^{\circ} \mathrm{C}$.

For proteomic analysis of interacting proteins, the magnetic beads were first washed with $50 \mu \mathrm{l}$ of $100 \mathrm{mM}$ TRIS pH 7.6. Subsequently, $50 \mu \mathrm{l}$ of $100 \mathrm{mM}$ TRIS $\mathrm{pH} 7.6$ containing $4 \mathrm{M}$ urea, $5 \mathrm{mM}$ dithiothreitol for reduction of disulfide bond, and $0.2 \mu \mathrm{g}$ of LysC for predigestion of proteins were added to each sample. After incubation of $3 \mathrm{~h}, 100 \mu \mathrm{l}$ of $100 \mathrm{mM}$ TRIS pH 7.6 and $10 \mathrm{mM}$ iodoacetamide were added for blocking of free cysteine side chains and samples were incubated in the dark for $5 \mathrm{~min}$. Samples were diluted with $100 \mu \mathrm{l}$ TRIS pH 7.6 to reduce the urea concentration and $1 \mu \mathrm{g}$ of trypsin was added to each sample. The samples were incubated for $14 \mathrm{~h}$ to complete protein digestion and subsequently trifluoroacetic acid was added to a final concentration of $0.5 \%$ to acidify the samples. Peptide mixture were separated from the magnetic beads before the desalting step. The beads were washed $2 \times$ with $75 \mu \mathrm{l}$ of $0.1 \%$ formic acid (FA) and the wash solvent was combined with the peptide mixtures. For sample desalting, three discs were stamped from C18 discs (Empore C18, 3 M) and placed into a $200 \mu$ pipette tip. Following binding of peptides, stage tips were washed $2 \times$ with $60 \mu \mathrm{l}$ of $0.1 \% \mathrm{FA}$ and peptides were eluted with $40 \%$ acetonitrile containing $30 \%$ methanol and $0.1 \% \mathrm{FA}$. Samples were dried in a speedvac and resuspended in $10 \mu \mathrm{l}$ of $0.1 \%$ FA. Peptide mixtures were analyzed by LC-MS/MS to identify and quantify proteins in all samples. First, peptides were separated by nano-reversed phase chromatography using a linear gradient from 2 to $35 \%$ acetonitrile over $50 \mathrm{~min}$ in $0.1 \% \mathrm{FA}$ on an inhouse-packed chromatography column in a nano-electrospray emitter tip. Eluting peptides were directly infused into the mass spectrometer (QExactive, Thermo Fisher) and detected in positive ionization mode. The operating cycle was programmed to detect peptides in the range from 300 to $1600 \mathrm{~m} / \mathrm{z}$ and up to 10 precursors were selected for MSMS analysis by CID fragmentation. Precursor ions required a charge state between +2 and +6 and a minimal signal intensity of $6 \times 10 \mathrm{e} 4$

Protein mapping and quantitative analysis raw LC-MS/MS data were searched against a C. glutamicum database retrieved from Uniprot (vs. 03/2017, 3093 protein entries) using a forward/reversed search by the Andromeda algorithm within the MaxQuant software suite. Peptides hits were searched with 17 p.p.m. precursor mass deviation in the first search and 3 p.p.m. for the main search. For MS/MS spectra, a mass accuracy of 25 p.p.m. was set. As variable modifications, acetylation of the protein $\mathrm{N}$-terminus, STY-phosphorylation, and methionine oxidation were selected. Carbamidomethylation of cysteine was the only fixed modification. Peptide match results were sorted by their probability score and filtered for $2 \%$ reversed peptide hits and $5 \%$ reversed protein hits.

To calculate protein enrichments and significance values, reversed protein hits and proteins with less than three quantitative values in any of the three sample types (control, mksB IP, and smc IP) were filtered out. The iBAQ-values were $\log 2$ transformed and median normalized. In case of one missing value in the triplicate measurements, the value was imputed using a closest neighbor method; for more missing data points, a random value from a standard distribution downshifted by a factor of 1.8 from the sample distribution and width of 0.3 was selected. Samples were compared using a Student's $t$-test, which was false discovery rate controlled by sample permutation.

Bacterial two-hybrid screening. Protein interactions obtained by mass spectrometry were confirmed via bacterial two-hybrid assays ${ }^{71}$, using compatible vectors expressing adenylate cyclase subunits T25 and T18 (pKT25/ pKNT25 and pUT18/ pUT18C). E. coli BTH101 co-transformed with respective vectors were plated on indicator medium LB/X-Gal (5-bromo-4-chloro-3-indolyl- $\beta$-D-galactopyranoside, $40 \mu \mathrm{g} / \mathrm{ml})$ supplemented with IPTG $(0.5 \mathrm{mM})$ and antibiotics kanamycin $(50 \mu \mathrm{g} / \mathrm{ml})$ carbenicillin $(100 \mu \mathrm{g} / \mathrm{ml})$, and streptomycin $(100 \mu \mathrm{g} / \mathrm{ml})$, and incubated at $30^{\circ} \mathrm{C}$ for $24 \mathrm{~h}$. Interacting hybrid proteins were identified by blue-white screening and $\beta$ galactosidase assays in a 96 -well plate format as previously described ${ }^{74}$. In brief, $0.1 \mathrm{ml}$ of overnight cultures of co-transformants were transferred to 96-well plates. Cells were pelleted and re-suspend in $80 \mu \mathrm{l}$ Z-buffer $\left(60 \mathrm{mM} \mathrm{Na}_{2} \mathrm{HPO}_{4}, 40 \mathrm{mM}\right.$

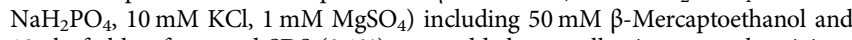
$10 \mu \mathrm{l}$ of chloroform and SDS $(0.1 \%)$ were added per well prior to gentle mixing. 
After centrifugation at $1000 \times g$ for $10 \mathrm{~min}$, clear lysates were transferred to a clean 96-well plate and $20 \mu \mathrm{l}$ of 2-Nitrophenyl $\beta$-D-galactopyranoside $(4 \mathrm{mg} / \mathrm{ml}$ in in Z-buffer) were added to each well at $30^{\circ} \mathrm{C}$. The reaction time of $\beta$-galactosidase activity was recorded until the addition of $30 \mu \mathrm{l}$ of $\mathrm{Na}_{2} \mathrm{CO}_{3}(1 \mathrm{M})$. Absorbance was determined at OD420 using a Tecan plate reader. Co-transformants harboring empty plasmids or pUT18C-zip/ pKT25-zip plasmids served as positive and negative controls. Miller units of negative controls served as reference and were set to zero. Miller units of any other sample were normalized accordingly. All C- and $\mathrm{N}$-terminal combinations of hybrid proteins were assayed and positive signals were confirmed through at least three replicates.

\section{Fluorescence microscopy. Fluorescence microscopy was performed with expo-} nentially grown cells mounted on agarose coated slides ( $1 \%$ agarose). Images were acquired on an Axio-Imager M1 fluorescence microscope (Carl Zeiss) with an EC Plan Neofluar $\times 100 / 1.3$ oil Ph3 objective and a 2.5x optovar. Fluorescence of protein fusions with eYFP (enhanced yellow fluorescent protein) and mCherry/ mCherry2 or DNA stained via Hoechst $33342(1 \mu \mathrm{g} / \mathrm{ml}$, Thermo Scientific) were detected using filter sets 46 HE YFP (EX BP 500/25, BS FT 515, and EM BP 535/ 30), 43 HE Cy 3 shift free (EX BP 550/25, BS FT 570, and EM BP 605/70), and 49 DAPI shift free (EX G 365, BS FT 395, and EM BP 445/50). Live-cell imaging and detection of fluorescently labeled condensin subunits were carried out using a Delta Vision Elite microscope (GE Healthcare, Applied Precision) with a standard four color InsightSSI module, a $\times 100 / 1.4$ oil PSF U-Plan S-Apo objective and the YFP (EX BP 513/17 and EM BP 548/22) and mCherry (EX BP 575/25 and EM BP 625/ 45) specific filter sets. To conduct time-lapse experiments, exponentially grown cells were diluted to an $\mathrm{OD}_{600}$ of 0.01 in $\mathrm{BHI}$ and loaded in a microfluidic chamber (B04A CellASIC ${ }^{\oplus}$, Onix); the environmental chamber was heated to $30^{\circ} \mathrm{C}$ and 0.75 psi were applied for nutrient supply throughout the experiment. Images were taken in $5 \mathrm{~min}$ intervals. For display of cellular fluorescence profiles sorted by cell length, FIJI and R software were utilized ${ }^{50,75,75,76}$.

ChIP combined with sequencing. Briefly, cells were crosslinked (1\% formaldehyde) for $30 \mathrm{~min}$ at room temperature (RT) and lysed. DNA was sheared by sonication, incubated with $\alpha$-mCherry antibody for $2 \mathrm{~h}$ at $4^{\circ} \mathrm{C}$, washed subsequently, and crosslinks were reverted at $65^{\circ} \mathrm{C}$. DNA purification was followed by library preparation and sequencing using an Illumina MiSeq system. Reads were aligned to the C. glutamicum ATCC 13032 genome sequence (GeneBankID: BX927147.1). Further data analysis was performed using online tools ${ }^{77}$. More in details, in vivo ChIP experiments with C. glutamicum ParB, SMC, or MksB proteins were conducted using strains with allelic replacements of respective proteins with mCherry-tagged versions. Exponentially growing cells were crosslinked in 1\% formaldehyde for $30 \mathrm{~min}$ at RT; for SMC- and MksB-mCherry ChIP experiments, cells were treated with Crosslink Gold (Diagenode) for $30 \mathrm{~min}$ at RT and washed twice in phosphate-buffered saline (PBS; $137 \mathrm{mM} \mathrm{NaCl}, 10 \mathrm{mM} \mathrm{Na}_{2} \mathrm{HPO}_{4}, 1.8 \mathrm{mM}$ $\mathrm{KH}_{2} \mathrm{PO}_{4}, 2.7 \mathrm{mM} \mathrm{KCl} \mathrm{pH} \mathrm{7.4.)} \mathrm{prior} \mathrm{to} \mathrm{formaldehyde} \mathrm{crosslinking.} \mathrm{Fixed} \mathrm{cells} \mathrm{were}$ subsequently washed in PBS and suspended in protoplast buffer $(50 \mathrm{mM}$ Tris $\mathrm{pH}$ 7.4, $50 \mathrm{mM} \mathrm{NaCl}, 10 \mathrm{mM}$ EDTA, $0.5 \mathrm{M}$ sucrose, EDTA-free protease inhibitor cocktail) supplemented with $20 \mathrm{mg} / \mathrm{ml}$ of lysozyme for $2 \mathrm{~h}$ at $37^{\circ} \mathrm{C}$. After washing in protoplast buffer, pellets were resuspended in buffer $\mathrm{L}(50 \mathrm{mM}$ HEPES-KOH $\mathrm{pH}$ $7.55,40 \mathrm{mM} \mathrm{NaCl}, 1 \mathrm{mM}$ EDTA, $1 \%$ Triton X-100, $0.1 \%$ deoxycholate, $0.1 \mathrm{mg} / \mathrm{ml}$ RNaseA; EDTA-free protease inhibitor cocktail) and DNA was sheared into fragments of around $800 \mathrm{bp}$ length by sonication using an ultrasonic cell disruptor (Branson Ultrasonics Sonifier ${ }^{\mathrm{m}} ; 20 \%$ amplitude, pulse $0.5 \mathrm{~s}$ on/off, $6 \times 20 \mathrm{~s}$ ). Following removal of cell debris $\left(20,000 \times g, 10 \mathrm{~min}, 4^{\circ} \mathrm{C}\right)$. Aliquots of cell extracts were stored for later use. Dynabeads ${ }^{\mathrm{mx}}$ Protein G (Thermo Fisher Scientific) were bound to an $\alpha$-mCherry Fantibody (BioVision, Inc.) in buffer $\mathrm{L}$ for $1.5 \mathrm{~h}$ at $4{ }^{\circ} \mathrm{C}$, washed in buffer $\mathrm{L}$, and subsequently incubated with cell extract for $2 \mathrm{~h}$ at $4^{\circ} \mathrm{C}$. Thereafter, beads were washed in buffer $\mathrm{L}$, in buffer L5 (50 mM HEPES-KOH pH $7.55,500 \mathrm{mM} \mathrm{NaCl}, 1 \mathrm{mM}$ EDTA, $1 \%$ Triton X-100, 0.1\% deoxycholate), in buffer

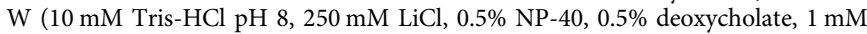
EDTA), and TE buffer (10 mM Tris-HCl pH 8, $1 \mathrm{mM}$ EDTA) consecutively and finally resuspended in TES buffer ( $10 \mathrm{mM}$ Tris- $\mathrm{HCl} \mathrm{pH} 8,10 \mathrm{mM}$ EDTA, $1 \%$ SDS). Extract samples were also supplemented with TES buffer and SDS to a final concentration of $1 \%$ SDS; crosslinks were reverted at $65^{\circ} \mathrm{C}$ overnight.

Phenol-chloroform extraction yielded DNA pellets, which were further purified using a DNA purification kit (QIAquick ${ }^{\circledast}$, Qiagen). qPCR was applied to confirm protein enrichment at specific chromosomal loci. Immunoprecipitation and extract samples were diluted 1:10 and 1:100 in water, yielding concentrations of $\sim 0.2-0.4 \mathrm{ng} / \mu \mathrm{l}$

For sequencing analyses, libraries of ChIP samples were prepared followed by sequencing utilizing an Illumina MiSeq system. Reads were aligned to the $\mathrm{C}$. glutamicum ATCC 13032 genome sequence (GeneBankID: BX927147.1), where RES167-specific genome deletions were manually cut using CLC Genomics Workbench. Data of extract and corresponding ChIP sample were each normalized based on read counts and the ratio of the number of reads per $0.5 \mathrm{~Kb}$ bin were determined via the Galaxy web platform 77,78 .

Real-time PCR. DNA amplification was performed using a $2 \times \mathrm{qPCR}$ Mastermix (KAPA SYBR ${ }^{\oplus}$ FAST, Peqlab) according to the manufacturer's instruction, where reaction volumes of $10 \mu \mathrm{l}$ contained $200 \mathrm{nM}$ oligonucleotides and $4 \mu \mathrm{l}$ of diluted DNA, respectively. Samples were measured in technical duplicates via an iQ5 multicolor real-time PCR detection system (Bio-Rad) and CT-values were determined via the Bio Rad-IQ"'5 software version 2.1. Primer efficiencies were estimated by calibration dilution curves and slope calculation ${ }^{79}$; data were analyzed via the $2^{-\mathrm{CCT}}$ method $^{80}$ accounting for dilution factors and sample volumes used for DNA purification. qPCR data of ChIP samples were normalized according to the ParB-mCherry2 signal obtained at locus parS1 in the wild-type background, serving as reference in each experiment.

Protein purification. ParB protein production was performed in E. coli BL21 pLysS via the pET-16b vector-based system. Cells were grown in $\mathrm{LB}$ at $37^{\circ} \mathrm{C}$; gene expression was induced adding $1 \mathrm{mM}$ IPTG following growth for $12 \mathrm{~h}$ at $18^{\circ} \mathrm{C}$. Subsequently, cells were suspended in washing buffer (50 mM Tris- $\mathrm{HCl} \mathrm{pH} \mathrm{7.4;}$ $100 \mathrm{mM} \mathrm{NaCl} ; 5 \mathrm{mM} ; \mathrm{MgCl}_{2} ; 1 \mathrm{mM}$ dithiothreitol) containing EDTA-free proteinase inhibitor (cOmplete ${ }^{\mathrm{TM}}$, Sigma) and DNAseI, and lysed using a high-pressure cell homogenizer. Cell debris and membranes were removed by centrifugation at $4{ }^{\circ} \mathrm{C}, 1700 \mathrm{~g}$ for $20 \mathrm{~min}$, and $150,000 \times g$ for $45 \mathrm{~min}$, respectively. Thereupon, batch purifications of His-tagged protein were performed under native conditions using Ni-NTA agarose (Protino ${ }^{\oplus}$, Macherey-Nagel) according to manufacturer's instruction. In brief, the equilibrated gel was incubated with clarified lysate for $60 \mathrm{~min}$ at $4{ }^{\circ} \mathrm{C}$ under gentle agitation and washed twice in washing buffer containing $80 \mathrm{mM}$ imidazole. Proteins were eluted in three steps using washing buffer with an imidazole concentration of $300 \mathrm{mM}$, concentrated via Amicon filter units (Merck) and further purified by applying size exclusion chromatography using an ÄKTApurifier system with a Superdex ${ }^{\mathrm{TM}} 200$ gel filtration column (GE Healthcare Life Sciences).

Electrophoretic mobility shift assay. DNA-ParB binding was assayed using purified protein and double-stranded DNA fragments of $\sim 1100 \mathrm{bp}$ length with or without two parS sites. Fragments were generated by PCRs of a C. glutamicum genomic locus surrounding parS9 and parS10 using primer pairs parS9mut-HindIII-up-F/parS10mut-EcoRI-D-R. ParB concentrations of 0.05-25 $\mu \mathrm{M}$ were incubated with $100 \mathrm{ng}$ DNA for $30 \mathrm{~min}$ at $30^{\circ} \mathrm{C}$, following sample separation in native gels (3-12\% polyacrylamide, ServaGel $\left.{ }^{\mathrm{TM}}\right)$. DNA was stained using SYBR ${ }^{\circledast}$ Green I (Invitrogen)

Photoactivated localization microscopy. C. glutamicum cells were fixed with $3 \%$ of formaldehyde prior to super-resolution imaging using a Zeiss ELYRA P.1 microscope with laser lines HR diode $50 \mathrm{~mW} 405 \mathrm{~nm}$ and HR DPSS $200 \mathrm{~mW}$ $561 \mathrm{~nm}$, and an Andor EMCCD iXon DU 897 camera. Cellular ParB-PAmCherry signals were further analyzed using Fiji software ${ }^{75}$ and identification of distinct protein clusters was carried out by applying the OPTICS algorithm in $\mathrm{R}^{49,50}$. For sample preparation, C. glutamicum cells expressing ParB-PAmCherry were collected in exponential growth phases, washed twice in PBS, and fixated in PBS + 3\% formaldehyde solution (36.5-38\% in $\mathrm{H}_{2} \mathrm{O}+10-15 \%$ methanol, Sigma Aldrich) for $30 \mathrm{~min}$ at $30^{\circ} \mathrm{C}$. Excess formaldehyde was subsequently quenched by adding $10 \mathrm{mM}$ glycine, cells were sedimented at $5000 \times g$ for $1 \mathrm{~min}$, resuspended in PBS containing $10 \mathrm{mM}$ glycine, and incubated for $5 \mathrm{~min}$ at RT. This quenching step was repeated three times; cells were finally diluted in buffer containing $50 \mathrm{mM}$ Tris $\mathrm{pH}$ $7.4,50 \mathrm{mM} \mathrm{NaCl}, 10 \mathrm{mM}$ EDTA, and $0.5 \mathrm{M}$ sucrose (TSEMS). Cells expressing MksB-PAmCherry and DivIVA-mNeonGreen were not fixed due to the low amount of MksB expressed (formaldehyde fixation renders part of the fluorophore population unable to fluoresce) but simply collected and washed in TSEMS buffer

Super-resolution imaging was performed on a Zeiss ELYRA P.1 microscope (laser lines HR diode $50 \mathrm{~mW} 405 \mathrm{~nm}$ and HR DPSS $200 \mathrm{~mW} 561 \mathrm{~nm}$ ). Cellular PAmCherry-tagged proteins were detected via an Andor EMCCD iXon DU 897 camera as described before, using a long-pass $570 \mathrm{~nm}$ filter (LP570) and an alpha Plan-Apochromat $\times 100 / 1.46$ Oil DIC M27 objective for imaging. Further, $100 \mathrm{~nm}$ TetraSpeck microspheres and the implemented drift correction tool served for drift correction; the Z-axis was stabilized via the "definite focus" system. PALM image calculation was performed applying the 2D $x / y$ Gaussian fit (Zenblack software, Zeiss) using a peak mask size of 9 pixels, where one pixel corresponds to $100 \mathrm{~nm}$ and a peak intensity to noise ratio of 6 . To exclude background and events resulting from the co-emission of co-localizing molecules, events were filtered for photon numbers between 70 and 350 , and PSF (point spread function) width at $1 / e$ maximum $(70-170 \mathrm{~nm})$ were applied. As a last step, events were grouped according to the following parameters: three on-frames with 0 off-frames allowed and a search radius of $30 \mathrm{~nm}$.

When imaging strains containing ParB-PAmCherry, four imaging series were taken for each field-of view, where each subsequent serie was characterized by a specific $405 \mathrm{~nm}$ laser linear gradient intensity range $(0.001 \%$ to $0.01 \%, 0.01 \%$ to $0.1 \%, 0.1 \%$ to $1 \%$, and $1 \%$ to $10 \%$ ). Every other imaging parameter remained the same in between the time series. The frame count for each collection was 10,000 frames and converted molecules were imaged using the $561 \mathrm{~nm}$ laser at $15 \%$ (transfer mode) for $50 \mathrm{~ms}$ at a 200 -fold EMCCD gain. For MksB-PAmCherry, the $405 \mathrm{~nm}$ laser linear gradient ranged between $0.005 \%$ and $0.5 \%$ within 5000 frames, while the other imaging parameters were kept the same as in the case of ParBPAmCherry. DivIVA-mNeonGreen was imaged for 10,000 frames using the 
$488 \mathrm{~nm}$ laser at $30 \%$ (transfer mode) for $50 \mathrm{~ms}$ at 200 EMCCD (electron multiplying charge-coupled device) gain. In this case no $405 \mathrm{~nm}$ laser was used, as the fluorophore is not photoactivatable. As the $488 \mathrm{~nm}$ laser causes activation of PAmCherry, MksB-PAmCherry was imaged prior than DivIVA-mNeongreen.

The workflow of protein cluster analysis is illustrated in Supplementary Fig. 7. The field-of-view in the bright-field channel was correct for illumination unevenness by dividing the field-of-view containing the cells of interest with an empty one (Process-Calculator Plus, Fiji) and enlarged ten times (bicubic interpolation). The resulting image was thresholded (Image-Adjust-Threshold) with default parameters and converted to a binary mask. A Fiji macro was then run on the binary mask to close the mask holes present within cells and to enlarge the cells mask themselves. Cells that were in contact with each other were separated via water shading. The perimeter coordinates corresponding to masks representing cells lying within the focus were extracted and used to exclude events originating from cells lying outside the focal plane and the background. The clustering structures of events within a cell were identified via the OPTICS algorithm in $\mathrm{R}^{49,50}$. OPTICS is a clustering algorithm based on two parameters: minimum points (MinPts-in this case, a point is an event) and epsilon ( $\varepsilon$-maximum search radius). As, in our case, only events within the same cell can belong to the same cluster, $\varepsilon$ was chosen so that it would include all the events present within each cell $(\varepsilon=3000 \mathrm{~nm})$. The effect of the MinPts value on the visualization of the clusterordering has been previously described ${ }^{49}$. Briefly, although the overall shape of the reachability plot does not differ greatly for different MinPts, a low value makes the reachability plot more jagged, while high values smoothen it (a high value also has the benefit to decrease the chance of "single-link" effects).

As we know from epifluorescence that ParB clusters are relatively big, we chose a MinPts value (32) that would smoothen minor density variability, while being able to identify strong event density variations (Supplementary Fig. 7). The resulting reachability plot showed the presence of multiple density peaks at different reachability distance supporting the idea that within each macrocluster there are subclusters. Reachability distance thresholds were then chosen to highlight such phenomenon. Specifically, 50 and $35 \mathrm{~nm}$ were chosen as thresholds, as, in the tested conditions, they were able to consistently identify and separate subclusters from the macroclusters they were lying into (Supplementary Fig. 7). Documentation is available at Github (https://github.com/GiacomoGiacomelli/ ParB-clustering-protein-profiling).

Chromosome conformation capture libraries. 3C/Hi-C libraries were generated as previously described by Val et al. ${ }^{48}$ with minor changes. Briefly, cells were grown in $200 \mathrm{ml}$ of $\mathrm{BHI}$ medium at $30^{\circ} \mathrm{C}$ to an $\mathrm{OD}_{600}$ of 3 and rediluted to a final concentration of $\sim 1 \times 10^{7} \mathrm{cells} / \mathrm{ml}$. Cells were crosslinked using fresh formaldehyde for $30 \mathrm{~min}$ at RT ( $3 \%$ final concentration; Sigma Aldrich Formalin $37 \%$ ) followed by $30 \mathrm{~min}$ at $4{ }^{\circ} \mathrm{C}$. Formaldehyde was quenched using a final concentration of $0.25 \mathrm{M}$ glycine for $20 \mathrm{~min}$ at RT. Cells were then collected by centrifugation, frozen in dry ice, and stored at $-80^{\circ} \mathrm{C}$ until use. Frozen pellets of $\sim 10^{9}$ cells were thawed on ice and suspended in a final volume of $1.1 \mathrm{~mL} 1 \times \mathrm{TE}$ ( $\mathrm{pH} 8$ ) and transferred in a VK01 Precellys Tube (beads beating). Fixed cells were disrupted using the following program on a precellys apparatus: 9 cycles $\times$ [20" -3500 r.p.m.; 30 " - pause]. Lysate was transferred to a $1.5 \mathrm{ml}$ tube, SDS $10 \%$ was added to the mix to a final concentration of $0.5 \%$ and the mix was incubated for $10 \mathrm{~min}$ at RT. $1 \mathrm{ml}$ of lysate was then transferred in a $5 \mathrm{ml}$ tube containing $4 \mathrm{ml}$ of digestion mix ( $1 \times$ NEB 3 buffer, $1 \%$ Triton X-100, and $1000 \mathrm{U}$ MluCI enzyme). DNA was digested for $3 \mathrm{~h}$ at $37^{\circ} \mathrm{C}$ under shaking. Insoluble fraction was then recovered through centrifugation $(16,000 \times g, 20 \mathrm{~min})$ and the obtained pellet was resuspended in $1 \mathrm{ml}$ of water and diluted in $15 \mathrm{ml}$ of ligation reaction mix ( $1 \times$ ligation buffer NEB without ATP, $1 \mathrm{mM}$ ATP, $0.1 \mathrm{mg} / \mathrm{ml}$ bovine serum albumin, 125 Units of T4 DNA ligase $5 \mathrm{U} / \mathrm{ml}$ ). Ligation was allowed to proceed for $4 \mathrm{~h}$ at $16^{\circ} \mathrm{C}$, followed by incubation overnight at $65^{\circ} \mathrm{C}$ in the presence of $250 \mathrm{mg} / \mathrm{ml}$ proteinase $\mathrm{K}, 0.5 \% \mathrm{SDS}$, and $5 \mathrm{mM}$ EDTA. Next morning, DNA was precipitated using $1 / 10$ th volume of $3 \mathrm{M} \mathrm{Na}$-Acetate ( $\mathrm{pH}$ 5.2) and one volume of isopropanol. After $1 \mathrm{~h}$ at $-80^{\circ} \mathrm{C}$, DNA was pelleted, resuspended in $900 \mu \mathrm{l} 1 \times$ TE buffer, and extracted with $900 \mu \mathrm{l}$ phenol-chloroform $\mathrm{pH}$ 8.0. DNA was again precipitated using 1/10th volume of $3 \mathrm{M} \mathrm{Na}$-Acetate ( $\mathrm{pH}$ 5.2) and 2.5 volume of cold Ethanol. Finally, DNA was resuspended in $100 \mu \mathrm{l} 1 \times \mathrm{TE}$ buffer supplemented with RNAse and incubated $30 \mathrm{~min}$ at $37^{\circ} \mathrm{C}$. $3 \mathrm{C}$ libraries were then processed as described ${ }^{48}$ and paired-end sequenced on an Illumina NextSeq apparatus $(2 \times 35 \mathrm{bp})$. DNA content per cell was further determined by flow cytometry, yielding an average number of six chromosome equivalents per cell for all strains analyzed by Hi-C like approaches (Supplementary Fig. 2B, C and Supplementary Table 2).

Contact map generation. Contact maps were generated as previously described ${ }^{38}$. Reads were aligned independently (forward and reverse) using Bowtie 2 in local and very sensitive mode and were assigned to a restriction fragment. Noninformative events (self-circularized DNA fragments, or uncut fragments) were discarded by taking into account the pair-reads relative directions and the distribution of the different configurations as described in Cournac et al. ${ }^{81}$. We then bin the genomes into regular units of $5 \mathrm{~Kb}$ to generate contact maps and normalized them using the sequential component normalization procedure ${ }^{81}$. Contact maps were then generated using Pyplot library and a saturation threshold at 99.5\% of the maximum value.

Contact map comparison. Ratio between contact maps was computed for each point of the map by dividing the amount of normalized contacts in one condition by the amount of normalized contacts in the other condition and by plotting the Log2 of the ratio. The color code reflects a decrease or increase of contacts in one condition compared to the other (blue or red signal, respectively). No change is represented by a white signal. To further compare the Hi-C data, we applied the HiCRep software ${ }^{56}$ at a resolution of $5 \mathrm{~Kb}$ with a smoothing index of 3 .

Identification of domains frontiers using directional index. To quantify the degree of directional preference, we applied on correlation matrices the same procedure as in Marbouty et al..$^{10}$. For each $5 \mathrm{~Kb}$ bin, we extracted the vector of interactions from the correlation matrix between the studied bin and bins at regular $5 \mathrm{~Kb}$ intervals, up to $250 \mathrm{~Kb}$ in left and right directions. The two vectors were then compared with a paired $t$-test to assess their statistical significant difference $(p=0.05)$. The directional preferences for the bin along the chromosome are represented as a bar plot with positive and negative tvalues shown as red and green bars.

Flow cytometry. DNA contents per cell were verified in C. glutamicum strains analyzed by $3 \mathrm{C}$ using flow cytometry as described before ${ }^{41}$ Flow cytometry analysis was performed as described before ${ }^{41}$. In short, exponentially growing cultures were treated with $25 \mu \mathrm{g} / \mathrm{ml}$ chloramphenicol for more than $4 \mathrm{~h}$, to induce replication runouts. Cells were fixated in 70\% ethanol $(1: 9 \mathrm{v} / \mathrm{v})$ and washed once in PBS. Cellular DNA was stained using SYBR ${ }^{\circledast}$ Green I (Invitrogen, 1:10,000 dilutions) for $15 \mathrm{~min}$ in the dark. Flow cytometry analysis was carried out subsequently using a BD Accuri C6 (BD Biosciences) equipped with a $488 \mathrm{~nm}$ laser. At least 200,000 events were collected per sample at a slow flow rate of $10 \mu \mathrm{l} / \mathrm{min}$ measuring $<5000$ events per second, applying an acquisition threshold of 650 set on the green channel FL1-H. Data analysis was performed using the BD Accuri C6 Plus software (BD Biosciences). At first, events derived from cell aggregates were identified in plots of SSC vs. width and excluded from DNA content analysis. Remaining events were plotted as histograms vs. DNA amount (FL1-A, EM BP 533/30) at log scale, where chromosome numbers were assigned in accordance to calibration standards described before ${ }^{41}$. All experiments were performed in biological triplicates.

Comparison of contact signals with transcriptional data. RNA-sequencing data for C. glutamicum were recovered from ENA (Project PRJEB4788). Only reads with a mapping quality above 30 were conserved. Raw signal was then binned to match the binning of the corresponding contact maps and plotted along the genome. Both contact and transcription signals were smoothed with a Savitzky-Golay filter as previously described in Lioy et al. ${ }^{38}$.

Statistics and reproducibility. Correlation coefficients, linear regressions and analyses of nearest neighbor distance distributions were calculated using Excel 2019, Graph Pad Prism (GraphPad Software), and R (R-studio v1.1.453, R version v3.5.0 $)^{50}$.

Micrographs contained in the main and supplementary figures are exemplary images from three biological replicates. Plasmid extractions in Fig. $5 \mathrm{f}$ were repeated three times; control western blottings in Supplementary Fig. 8A were performed once, and gels in Supplementary Fig. 12B are exemplary data from two replicates.

Reporting summary. Further information on research design is available in the Nature Research Reporting Summary linked to this article.

\section{Data availability}

C. glutamicum ATCC 13032 genome sequence was obtained from GeneBank (GeneBankID: BX927147.1). RNA-seq data for C. glutamicum were recovered from ENA (Project PRJEB4788). Proteomic data are available via ProteomeXchange with the project identifier PXD008916 (ref. ${ }^{82}$ ). Genome-wide sequencing reads of ChIP-seq and chromosome conformation capture assays generated in this study are available in the Sequence Read Archive (SRA) under accession numbers PRJNA529385 and PRJNA525583. Flow cytometry raw data results were deposited in the FlowRepository database (accession number FR-FCM-Z2DJ) ${ }^{80}$. The source data underlying Figs. $2 c$, h, $3 \mathrm{~b}, \mathrm{~d}, \mathrm{e}$, and 5b, c, e, f and Supplementary Figs. 2B, C, 3B-D, 5A, D, 7C-E, 8A-C, 9B-D, $10 \mathrm{~A}, \mathrm{~B}, \mathrm{D}, 12 \mathrm{~A}, \mathrm{~B}, \mathrm{D}$, and $13 \mathrm{~A}, \mathrm{C}$ are provided as a Source Data file.

\section{Code availability}

The custom computer algorithm used in the analysis of PALM data has been deposited in Github (https://github.com/GiacomoGiacomelli/ParB-clustering-protein-profiling).

Received: 2 May 2019; Accepted: 21 February 2020; Published online: 20 March 2020 


\section{References}

1. Sazer, S. \& Schiessel, H. The biology and polymer physics underlying largescale chromosome organization. Traffic 19, 87-104 (2018).

2. Badrinarayanan, A., Le, T. B. K. \& Laub, M. T. Bacterial chromosome organization and segregation. Annu. Rev. Cell Dev. Biol. 31, 171-199 (2015).

3. Gruber, S. Multilayer chromosome organization through DNA bending, bridging and extrusion. Curr. Opin. Microbiol. 22, 102-110 (2014).

4. Kleckner, N. et al. The bacterial nucleoid: nature, dynamics and sister segregation. Curr. Opin. Microbiol. 22, 127-137 (2014).

5. Livny, J., Yamaichi, Y. \& Waldor, M. K. Distribution of centromere-like parS sites in bacteria: insights from comparative genomics. J. Bacteriol. 189, 8693-8703 (2007).

6. Rodionov, O., Lobocka, M. \& Yarmolinsky, M. Silencing of genes flanking the P1 plasmid centromere. Science 283, 546-549 (1999).

7. Murray, H., Ferreira, H. \& Errington, J. The bacterial chromosome segregation protein Spo0J spreads along DNA from parS nucleation sites. Mol. Microbiol. 61, 1352-1361 (2006).

8. Graham, T. G. W. et al. ParB spreading requires DNA bridging. Genes Dev. 28, 1228-1238 (2014).

9. Broedersz, C. P. et al. Condensation and localization of the partitioning protein ParB on the bacterial chromosome. Proc. Natl Acad. Sci. USA 111, 8809-8814 (2014).

10. Marbouty, M. et al. Condensin- and replication-mediated bacterial chromosome folding and origin condensation revealed by $\mathrm{Hi}-\mathrm{C}$ and superresolution imaging. Mol. Cell 59, 588-602 (2015).

11. Sanchez, A. et al. Stochastic self-assembly of ParB proteins builds the bacterial DNA segregation apparatus. Cell Syst. 1, 163-173 (2015).

12. Leonard, T. A., Butler, P. J. \& Löwe, J. Bacterial chromosome segregation: structure and DNA binding of the Soj dimer - a conserved biological switch. EMBO J. 24, 270-282 (2005).

13. Zhang, H. \& Schumacher, M. A. Structures of partition protein ParA with nonspecific DNA and ParB effector reveal molecular insights into principles governing Walker-box DNA segregation. Genes Dev. 31, 481-492 (2017).

14. Vecchiarelli, A. G., Hwang, L. C. \& Mizuuchi, K. Cell-free study of F plasmid partition provides evidence for cargo transport by a diffusion-ratchet mechanism. Proc. Natl Acad. Sci. USA 110, E1390-E1397 (2013).

15. Vecchiarelli, A. G., Neuman, K. C. \& Mizuuchi, K. A propagating ATPase gradient drives transport of surface-confined cellular cargo. Proc. Natl Acad. Sci. USA 111, 4880-4885 (2014).

16. Hu, L., Vecchiarelli, A. G., Mizuuchi, K., Neuman, K. C. \& Liu, J. Directed and persistent movement arises from mechanochemistry of the ParA/ParB system. Proc. Natl Acad. Sci. USA 112, E7055-E7064 (2015).

17. Surovtsev, I. V., Campos, M. \& Jacobs-Wagner, C. DNA-relay mechanism is sufficient to explain ParA-dependent intracellular transport and patterning of single and multiple cargos. Proc. Natl Acad. Sci. USA 113, E7268-E7276 (2016).

18. Lagage, V., Boccard, F. \& Vallet-Gely, I. Regional control of chromosome segregation in Pseudomonas aeruginosa. PLOS Genet. 12, e1006428 (2016).

19. Tran, N. T. et al. Permissive zones for the centromere-binding protein ParB on the Caulobacter crescentus chromosome. Nucleic Acids Res. 46, 1196-1209 (2018).

20. Sullivan, N. L., Marquis, K. A. \& Rudner, D. Z. Recruitment of SMC by ParBparS organizes the origin region and promotes efficient chromosome segregation. Cell 137, 697-707 (2009).

21. Wang, X. et al. Condensin promotes the juxtaposition of DNA flanking its loading site in Bacillus subtilis. Genes Dev. 29, 1661-1675 (2015)

22. Tran, N. T., Laub, M. T. \& Le, T. B. K. SMC progressively aligns chromosomal arms in Caulobacter crescentus but is antagonized by convergent transcription. Cell Rep. 20, 2057-2071 (2017)

23. Cobbe, N. \& Heck, M. M. The evolution of SMC proteins: phylogenetic analysis and structural implications. Mol. Biol. Evol. 21, 332-347 (2004).

24. Niki, H., Jaffe, A., Imamura, R., Ogura, T. \& Hiraga, S. The new gene mukB codes for a $177 \mathrm{kd}$ protein with coiled-coil domains involved in chromosome partitioning of E. coli. EMBO J. 10, 183-193 (1991).

25. Britton, R. A., Lin, D. C. \& Grossman, A. D. Characterization of a prokaryotic SMC protein involved in chromosome partitioning. Genes Dev. 12, 1254-1259 (1998).

26. Jensen, R. B. \& Shapiro, L. The Caulobacter crescentus $s m c$ gene is required for cell cycle progression and chromosome segregation. Proc. Natl Acad. Sci. USA 96, 10661-10666 (1999).

27. Bürmann, F. et al. An asymmetric SMC-kleisin bridge in prokaryotic condensin. Nat. Struct. Mol. Biol. 20, 371 (2013).

28. Diebold-Durand, M. L. et al. Structure of full-length SMC and rearrangements required for chromosome organization. Mol. Cell 67, 334-347.e335 (2017).

29. Terakawa, T. et al. The condensin complex is a mechanochemical motor that translocates along DNA. Science 358, 672-676 (2017).

30. Ganji, M. et al. Real-time imaging of DNA loop extrusion by condensin. Science 360, 102-105 (2018).
31. Wang, X., Brandão, H. B., Le, T. B. K., Laub, M. T. \& Rudner, D. Z. Bacillus subtilis SMC complexes juxtapose chromosome arms as they travel from origin to terminus. Science $355,524-527$ (2017).

32. Minnen, A. et al. Control of Smc coiled coil architecture by the ATPase heads facilitates targeting to chromosomal ParB/parS and release onto flanking DNA. Cell Rep. 14, 2003-2016 (2016).

33. Wang, X. et al. In vivo evidence for ATPase-dependent DNA translocation by the Bacillus subtilis SMC condensin complex. Mol. Cell 71, 841-847.e845 (2018).

34. Minnen, A., Attaiech, L., Thon, M., Gruber, S. \& Veening, J. W. SMC is recruited to oriC by $\mathrm{ParB}$ and promotes chromosome segregation in Streptococcus pneumoniae. Mol. Microbiol. 81, 676-688 (2011).

35. Gruber, S. \& Errington, J. Recruitment of condensin to replication origin regions by $\mathrm{ParB} / \mathrm{SpoOJ}$ promotes chromosome segregation in B. subtilis. Cell 137, 685-696 (2009).

36. Le, T. B., Imakaev, M. V., Mirny, L. A. \& Laub, M. T. High-resolution mapping of the spatial organization of a bacterial chromosome. Science 342, 731-734 (2013)

37. Wang, X., Liu, X., Possoz, C. \& Sherratt, D. J. The two Escherichia coli chromosome arms locate to separate cell halves. Genes Dev. 20, 1727-1731 (2006).

38. Lioy, V. S. et al. Multiscale structuring of the E. coli chromosome by nucleoidassociated and condensin proteins. Cell 172, 771-783.e718 (2018).

39. Nolivos, S. et al. MatP regulates the coordinated action of topoisomerase IV and MukBEF in chromosome segregation. Nat. Commun. 7, 10466-10466 (2016).

40. Petrushenko, Z. M., She, W. \& Rybenkov, V. V. A new family of bacterial condensins. Mol. Microbiol. 81, 881-896 (2011).

41. Böhm, K. et al. Novel chromosome organization pattern in Actinomycetalesoverlapping replication cycles combined with diploidy. $m$ Bio $\mathbf{8}$, e00511-e00517 (2017)

42. Donovan, C., Schwaiger, A., Krämer, R. \& Bramkamp, M. Subcellular localization and characterization of the ParAB system from Corynebacterium glutamicum. J. Bacteriol. 192, 3441-3451 (2010).

43. Donovan, C., Sieger, B., Krämer, R. \& Bramkamp, M. A synthetic Escherichia coli system identifies a conserved origin tethering factor in Actinobacteria. Mol. Microbiol. 84, 105-116 (2012).

44. Ireton, K., Gunther, N. W. \& Grossman, A. D. spo0J is required for normal chromosome segregation as well as the initiation of sporulation in Bacillus subtilis. J. Bacteriol. 176, 5320-5329 (1994).

45. Lee, P. S. \& Grossman, A. D. The chromosome partitioning proteins Soj (ParA) and Spo0J (ParB) contribute to accurate chromosome partitioning, separation of replicated sister origins, and regulation of replication initiation in Bacillus subtilis. Mol. Microbiol. 60, 853-869 (2006).

46. Donovan, C., Schauss, A., Krämer, R. \& Bramkamp, M. Chromosome segregation impacts on cell growth and division site selection in Corynebacterium glutamicum. PLoS ONE 8, e55078 (2013).

47. Dekker, J., Rippe, K., Dekker, M. \& Kleckner, N. Capturing chromosome conformation. Science 295, 1306-1311 (2002).

48. Val, M.-E. et al. A checkpoint control orchestrates the replication of the two chromosomes of Vibrio cholerae. Sci. Adv. 2, e1501914 (2016).

49. Ankerst, M., Breunig, M. M., Kriegel, H. \& Sander, J. OPTICS: ordering points to identify the clustering structure. ACM SIGMOD Record 2, 49-60 (1999).

50. R Core Team. R: a langauge and environment for statistical computing. $R$ Foundation Statistical Computing http://www.R-project.org/ (2014).

51. Lenarcic, R. et al. Localisation of DivIVA by targeting to negatively curved membranes. EMBO J. 28, 2272-2282 (2009)

52. Letek, M. et al. DivIVA is required for polar growth in the MreB-lacking rodshaped actinomycete Corynebacterium glutamicum. J. Bacteriol. 190, 3283-3292 (2008)

53. Schwartz, M. A. \& Shapiro, L. An SMC ATPase mutant disrupts chromosome segregation in Caulobacter. Mol. Microbiol. 82, 1359-1374 (2011)

54. Hirano, M. \& Hirano, T. Positive and negative regulation of SMC-DNA interactions by ATP and accessory proteins. EMBO J. 23, 2664-2673 (2004).

55. Hirano, M. \& Hirano, T. Opening closed arms: long-distance activation of SMC ATPase by hinge-DNA interactions. Mol. Cell 21, 175-186 (2006).

56. Yang, T. et al. HiCRep: assessing the reproducibility of Hi-C data using a stratum-adjusted correlation coefficient. Genome Res. 27, 1939-1949 (2017).

57. Eikmanns, B. J., Kleinertz, E., Liebl, W. \& Sahm, H. A family of Corynebacterium glutamicum/Escherichia coli shuttle vectors for cloning, controlled gene expression, and promoter probing. Gene 102, 93-98 (1991)

58. Cremer, J., Eggeling, L. \& Sahm, H. Cloning the dapA dapB cluster of the lysine-secreting bacterium Corynebacterium glutamicum. Mol. Gen. Genet. 220, 478-480 (1990).

59. Kirchner, O. \& Tauch, A. Tools for genetic engineering in the amino acidproducing bacterium Corynebacterium glutamicum. J. Biotechnol. 104, 287-299 (2003) 
60. Reinscheid, D. J., Kronemeyer, W., Eggeling, L., Eikmanns, B. J. \& Sahm, H. Stable expression of hom-1-thrB in Corynebacterium glutamicum and its effect on the carbon flux to threonine and related amino acids. Appl. Environ. Microbiol. 60, 126-132 (1994).

61. Hassler, M., Shaltiel, I. A. \& Haering, C. H. Towards a unified model of SMC complex function. Curr. Biol. 28, R1266-R1281 (2018).

62. Doron, S. et al. Systematic discovery of antiphage defense systems in the microbial pangenome. Science 359, eaar4120 (2018).

63. Sota, M. et al. Shifts in the host range of a promiscuous plasmid through parallel evolution of its replication initiation protein. ISME J. 4, 1568 (2010).

64. Maestro, B., Sanz, J. M., Díaz-Orejas, R. \& Fernández-Tresguerres, E. Modulation of pPS10 host range by plasmid-encoded RepA initiator protein. J. Bacteriol. 185, 1367-1375 (2003).

65. De Gelder, L., Ponciano, J. M., Joyce, P. \& Top, E. M. Stability of a promiscuous plasmid in different hosts: no guarantee for a long-term relationship. Microbiology 153, 452-463 (2007).

66. Roth, S. et al. Rad50-CARD9 interactions link cytosolic DNA sensing to IL-1 $\beta$ production. Nat. Immunol. 15, 538 (2014).

67. Aragón, L. The Smc5/6 complex: new and old functions of the enigmatic longdistance relative. Annu. Rev. Genet. 52, 89-107 (2018).

68. Badrinarayanan, A., Reyes-Lamothe, R., Uphoff, S., Leake, M. C. \& Sherratt, D. J. In vivo architecture and action of bacterial structural maintenance of chromosome proteins. Science 338, 528-531 (2012).

69. Woo, J. S. et al. Structural studies of a bacterial condensin complex reveal ATP-dependent disruption of intersubunit interactions. Cell 136, 85-96 (2009).

70. Debaugny, R. E. et al. A conserved mechanism drives partition complex assembly on bacterial chromosomes and plasmids. Mol. Syst. Biol. 14, e8516 (2018).

71. Karimova, G., Pidoux, J., Ullmann, A. \& Ladant, D. A bacterial two-hybrid system based on a reconstituted signal transduction pathway. Proc. Natl Acad. Sci. USA 95, 5752-5756 (1998).

72. Schäfer, A. et al. Small mobilizable multi-purpose cloning vectors derived from the Escherichia coli plasmids pK18 and pK19: selection of defined deletions in the chromosome of Corynebacterium glutamicum. Gene 145 69-73 (1994).

73. Keilhauer, C., Eggeling, L. \& Sahm, H. Isoleucine synthesis in Corynebacterium glutamicum: molecular analysis of the $i l v B$-ilvN-ilvC operon. J. Bacteriol. 175, 5595-5603 (1993)

74. Mehla, J., Caufield, J. H., Sakhawalkar, N. \& Uetz, P. A comparison of twohybrid approaches for detecting protein-protein interactions. Methods Enzymol. 586, 333-358 (2017).

75. Schindelin, J. et al. Fiji: an open-source platform for biological-image analysis. Nat. Methods 9, 676-682 (2012).

76. Ducret, A., Quardokus, E. M. \& Brun, Y. V. MicrobeJ, a tool for high throughput bacterial cell detection and quantitative analysis. Nat. Microbiol. 1, 16077 (2016)

77. Ramírez, F. et al. deepTools2: a next generation web server for deepsequencing data analysis. Nucleic Acids Res. 44, W160-W165 (2016).

78. Afgan, E. et al. The Galaxy platform for accessible, reproducible and collaborative biomedical analyses: 2016 update. Nucleic Acids Res. 44, W3-W10 (2016).

79. Rasmussen, R. in Rapid Cycle Real-Time PCR Methods and Applications (eds Wittwer, C., Meuer, S. \& Nakagawara, K.) 21-34 (Springer, 2001).

80. Livak, K. J. \& Schmittgen, T. D. Analysis of relative gene expression data using real-time quantitative PCR and the 2(-Delta Delta $\mathrm{C}(\mathrm{T}))$ method. Methods 25, 402-408 (2001).

81. Cournac, A., Marie-Nelly, H., Marbouty, M., Koszul, R. \& Mozziconacci, J. Normalization of a chromosomal contact map. BMC Genomics 13, 436 (2012).
82. Deutsch, E. W. et al. The ProteomeXchange consortium in 2017: supporting the cultural change in proteomics public data deposition. Nucleic Acids Res. 45, D1100-D1106 (2017).

\section{Acknowledgements}

This work was funded by grants to M.B. from the Deutsche Forschungsgemeinschaft (project 326464754 in TRR174 and BR2815/6-2) and the Ministry of Science and Education (BMBF: 031A302 e:Bio-Modul II: 0.6 plus). This research was supported by funding to R.K. from the European Research Council under the Horizon 2020 Program (ERC grant agreement 260822), and from Agence Nationale pour la Recherche (HiResBac ANR-15-CE11-0023-03) and JPI-EC-AMR STARCS ANR-16-JPEC-0003-05 all to R.K. We thank our colleagues from R.K. and M.B. labs for discussions, feedback, and comments on the manuscripts, and the interpretation of the data. We are thankful to Professor Dr Stephan Gruber (Department of Fundamental Microbiology, University of Lausanne) for inspiring discussions and for his help with ChIP-seq. We are grateful to Dr Andreas Brachmann (LMU) for his help with the genome sequencing.

\section{Author contributions}

Conceptualization: K.B., M.M., R.K., and M.B. Methodology-Strain constructions and analyses, epifluorescence microscopy, ChIP, bacterial two-hybrid screening, in-vitro protein assays: K.B.; Chromosome conformation capture: M.M.; PALM: G.G.; Mass spectrometry: A.S. and A.I. Writing manuscript: K.B., M.M., A.S. (Methods), G.G. (Methods), R.K., and M.B.

\section{Competing interests}

The authors declare no competing interests.

\section{Additional information}

Supplementary information is available for this paper at https://doi.org/10.1038/s41467020-15238-4.

Correspondence and requests for materials should be addressed to M.M. or M.B.

Peer review information Nature Communications thanks Hugo Brandão and the other, anonymous, reviewers for their contribution to the peer review of this work.

Reprints and permission information is available at http://www.nature.com/reprints

Publisher's note Springer Nature remains neutral with regard to jurisdictional claims in published maps and institutional affiliations.

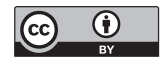

Open Access This article is licensed under a Creative Common Attribution 4.0 International License, which permits use, sharing, adaptation, distribution and reproduction in any medium or format, as long as you give appropriate credit to the original author(s) and the source, provide a link to the Creative Commons license, and indicate if changes were made. The images or other third party material in this article are included in the article's Creative Commons license, unless indicated otherwise in a credit line to the material. If material is not included in the article's Creative Commons license and your intended use is not permitted by statutory regulation or exceeds the permitted use, you will need to obtain permission directly from the copyright holder. To view a copy of this license, visit http://creativecommons.org/ licenses/by/4.0/

(C) The Author(s) 2020 